\title{
Bearing-based Network Localizability: a Unifying View
}

\author{
Federica Arrigoni and Andrea Fusiello
}

\begin{abstract}
This paper provides a unifying view and offers new insights on bearing-based network localizability, that is the problem of establishing whether a set of directions between pairs of nodes uniquely determines (up to translation and scale) the position of the nodes in d-space. If nodes represent cameras then we are in the context of global structure from motion. The contribution of the paper is theoretical: first, we rewrite and link in a coherent structure several results that have been presented in different communities using disparate formalisms; second, we derive some new localizability results within the edge-based formulation.
\end{abstract}

Index Terms—bearing-based localization; direction-based localization; parallel rigidity; bearing rigidity; structure from motion

\section{INTRODUCTION}

Bearing-based (or direction-based) network localization is a fundamental problem in many computing and networking tasks. The goal is to recover the position of $n$ nodes in d-space (with $n \geqslant 2$ and $d \geqslant 2$ ), given a redundant set of (possibly noisy) directions between pairs of nodes. Each node can represent any sensor able to measure the direction of the line joining its location to that of its neighbors (see [1], [2], [3]). One example is a network of cameras [4], in which case we are dealing with global structure from motion [5]. The problem can be profitably modeled by introducing a graph $\mathcal{G}=(\mathcal{V}, \mathcal{E})$ where vertices are the sensors and edges correspond to the available measures. Existing methods (e.g. [6], [7], [8], [9]) differ in the problem formulation (deterministic versus probabilistic) and the computational model (centralized versus distributed).

A fundamental question concerns the localizability of the network - which is the main focus of this paper, namely the problem of establishing whether bearing-based network localization is well-posed. Clearly, node locations can not be absolutely determined, since translations and dilations of a solution yield the same directions, and hence produce other solutions. Thus the question is whether the measures uniquely determine (up to translation and scale) the node locations, i.e., after fixing the position of two nodes (which essentially fixes the global translation and scale), all the other nodes are uniquely defined by the directions. Requiring that $\mathcal{G}$ is connected is not sufficient to guarantee the uniqueness of the solution, but more complicated assumptions are required, which are studied under the name of parallel rigidity (or bearing rigidity).

Several theoretical results about parallel rigidity are present in the literature [9], [10], [11], [12], [13], [14], [15], [16], [17], [18], [19], as well as practical algorithms for finding maximal subgraphs in which the localization problem is well-posed [20], [21], [22]. These works come from disparate

- F. Arrigoni and A. Fusiello are with the DPIA, University of Udine, Italy. Email:arrigoni.federica@spes.uniud.it, andrea.fusiello@uniud.it

- F. Arrigoni is currently with the Czech Technical University in Prague communities: discrete geometry [10], [11], [12], [18], [22]; computer vision [14], [19]; robotics [21]; decision and control [9], [15], [16], [17], [20]; computer-aided design [13].

The rigidity question can be posed either in terms of a point formation [15], [17], that is a configuration of $n$ nodes in d-space, or in terms of the underlying graph $\mathcal{G}=(\mathcal{V}, \mathcal{E})$ without reference to the specific values of the node locations [12], under the assumption that they are generic. The former gives rise to an algebraic characterization of localizability in terms of the rank of specific matrices derived from the coordinates of the nodes [9], [14], [16], [19], whereas the latter makes a combinatorial characterization of parallel rigidity possible [10], [11], [13]. A related concept is that of parallel rigidity index [18] in which the directions (instead of node locations) are assumed to be generic.

In this paper we consider the absolute version of the localizability problem, namely we assume that all the directions are expressed in a common rotational reference frame. See [23] for the relative version of the problem, where no global coordinate frame is known. We also assume that the network is anchor free, i.e., all the nodes have unknown positions. Some localizability results in the presence of nodes whose position is known in advance (anchors), are reported in [9].

A related topic, which is not covered in this paper, is distance-based network localization [24], [25], where each node can measure relative distances to a set of other nodes. The corresponding theory on the uniqueness of the solution is known as classical rigidity [26], which characterizes wellposed instances of the localization problem in 2-space. However, no such characterization has been shown to hold for $d \geqslant 3$. Parallel rigidity, instead, has a simpler structure since the direction constraints between pairs of nodes can be expressed as linear equations, which allows to solve the localizability problem for all $d$.

\subsection{Problem Definition}

Consider a network consisting of $n$ nodes labeled from 1 to $n$, where each node is located at a fixed (unknown) position in $\mathbb{R}^{d}$, and suppose that some pairs of nodes can measure 
the direction of the line joining their locations. Let $\mathbf{p}_{i} \in \mathbb{R}^{d}$ denote the location of node $i$ and let

$$
\mathbf{u}_{i j}=\frac{\mathbf{p}_{j}-\mathbf{p}_{i}}{\left\|\mathbf{p}_{j}-\mathbf{p}_{i}\right\|}
$$

denote the direction between node $i$ and node $j$, where $\|\cdot\|$ denotes the Euclidean norm in $\mathbb{R}^{d}$. In some applications the sign of the directions may be unspecified, in which case $\pm \mathbf{u}_{i j}$ is known. The neighbor relationships of the network can be represented as a simple directed graph $\mathcal{G}=(\mathcal{V}, \mathcal{E})$ with vertex set $\mathcal{V}=\{1,2, \ldots, n\}$ and edge set $\mathcal{E}$ such that $(i, j) \in \mathcal{E}$ if there is a measured direction between node $i$ and node $j$, with $m=|\mathcal{E}|$. We do not assume full measurements, i.e. $\mathcal{G}$ may not be complete.

The $d$-dimensional bearing-based network localization problem consists in determining the node locations $\mathbf{p}_{1}, \ldots, \mathbf{p}_{n}$ in $\mathbb{R}^{d}$, given the (redundant) set of pairwise measures $\left\{\mathbf{u}_{i j}\right\}_{(i, j) \in \mathcal{E}}$, as shown in Figure 1. These measures may be corrupted by noise, in which case the goal is to combine them into an estimate of the locations where errors are mitigated by exploiting the redundancy in the data.

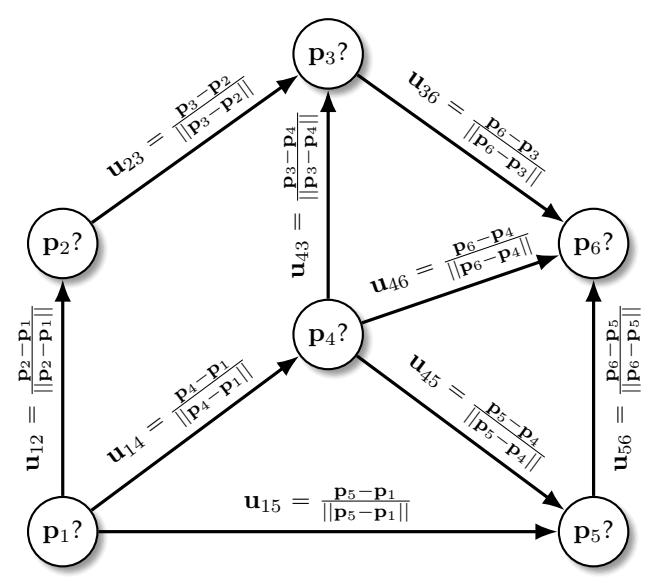

Figure 1: The bearing-based localization problem.

Given a set of (noise-free) directions and the underlying graph, the unknown node locations $\mathbf{p}_{i} \in \mathbb{R}^{d}$ can be recovered as the solution of the following system

$$
\mathbf{p}_{j}-\mathbf{p}_{i}=\alpha_{i j} \mathbf{u}_{i j} \quad(i, j) \in \mathcal{E}
$$

where $\alpha_{i j} \in \mathbb{R}$ are unknown as well.

Let $P$ be the $d \times n$ matrix obtained by juxtaposing all the node locations, namely $P=\left[\mathbf{p}_{1} \ldots \mathbf{p}_{n}\right]$, let $U$ be the $d \times m$ matrix obtained by juxtaposing all the edge directions, namely $U=\left[\begin{array}{llll}\mathbf{u}_{12} & \ldots & \mathbf{u}_{i j} & \ldots\end{array}\right]$, and let $\boldsymbol{\alpha} \in \mathbb{R}^{m}$ be the vector containing all the scales $\alpha_{i j}$. It is easy to see that the equations above can be expressed in matrix form as

$$
P B=U \operatorname{diag}(\boldsymbol{\alpha})
$$

where $B$ denotes the $n \times m$ incidence matrix of $\mathcal{G}$, which is defined in Equation (80), and $\operatorname{diag}(\boldsymbol{\alpha})$ produces a diagonal matrix with elements $\alpha_{i j}$ along the diagonal.

Applying the vectorization operator $\operatorname{vec}(\cdot)$ to both sides in (3) and using formulas (90) and (95) we get

$$
\left(B^{\top} \otimes I_{d}\right) \mathbf{p}=\left(I_{m} \odot U\right) \boldsymbol{\alpha}
$$

where $\mathbf{p}=\operatorname{vec}(P), \otimes$ denotes the Kronecker product, $\odot$ denotes the Khatri-Rao product, and $I_{d}$ (respectively $I_{m}$ ) denotes the $d \times d$ (respectively $m \times m$ ) identity matrix. See Equations (86) and (93) for the definitions of such matrix products.

We are interested here in establishing whether Equation (4) admits a unique solution (up to translation and scale). Note that both $\mathbf{p} \in \mathbb{R}^{d n}$ and $\boldsymbol{\alpha} \in \mathbb{R}^{m}$ are unknown. There are two paths that can be followed in order to remove one unknown: node-based [9], [10], [11], [12], [13], [15], [16], [17], [19], [21] and edge-based [14], [18], [20], [22]. The former derives a system of equations in terms of the node locations only, whereas the latter reduces (4) to a system of equations with the sole scales as unknowns. The node-based formulation is better studied than the edge-based one, which is fairly recent.

\subsection{Contribution}

In this paper - for the first time in the literature - we provide a unifying view of bearing-based localizability, rewriting results proposed in different scenarios using the same theoretical formalism, while at the same time also proposing some novel results which fill in gaps of knowledge, particularly related to the edge-based formulation. Note that some of these results were previously announced in our conference paper [14]. More precisely, our contributions are the following.

First, we provide a comprehensive survey on node-based parallel rigidity, which is reported in Section 2, considering both the standard definition of parallel rigidity, which involves a formation of $n$ nodes in d-space, and the concept of generic parallel rigidity, which is a property of the graph $\mathcal{G}=(\mathcal{V}, \mathcal{E})$. A novel result linking rigidity in $\mathbb{R}^{d}$ with rigidity in $\mathbb{R}^{d+1}$ is also derived (Proposition 2).

Secondly, as our main contribution, in Section 3 we describe the edge-based formulation of parallel rigidity, which builds upon [14], [20], [22]. We show that this formulation is theoretically equivalent to the node-based one (Proposition 4), it entails a compact matrix formulation (Theorem 7), and it also enables us to prove results involving the structure of the graph. Specifically, we prove that biconnectivity is necessary for parallel rigidity (Proposition 7) and we derive sufficient conditions for parallel rigidity (Theorems 9 and 10) based on the existence of cycle bases of $\mathcal{G}$ with certain properties.

Then, in Section 4 we rewrite in simpler terms the concept of parallel rigidity index defined in [18], providing its direct computation for some simple cases (Proposition 10 and Corollary 3) and explaining how it relates to parallel rigidity. We also underline its impact on bearing-based network localization, specifically we point out which graphs are better suited for the localization problem since they promote error compensation in the presence of noise.

Finally, in Section 5 we explain how bearing-based localizability is related to the structure from motion problem in Computer Vision, and we provide some examples of datasets encountered in real scenarios where the localization problem is not well posed.

The theory presented in this paper requires some basic notions from graph theory, which are covered in Ap- 
pendix $\mathrm{A}$, and the definitions of the Kronecker and KhatriRao products, which are given in Appendix B, included as additional material.

\section{Node-based Parallel Rigidity}

The theory of parallel rigidity is concerned with the problem of establishing if there are enough direction constraints (and they are distributed well enough) to ensure that all the feasible solutions to bearing-based network localization differ by translation and scale. The node-based formulation of parallel rigidity - which is the classical way to study the solvability of the localization problem - reasons in terms of node positions, and it is based on the concept of point formation. A complete treatment of this subject can be found in [10], [11], [13], [15], [16], [17].

\subsection{Rigidity of a Point Formation}

Let us start with the definition of point formation.

Definition 1. A $d$-dimensional point formation (or embedding) $\mathcal{F}_{\mathbf{p}}$ is a set $\mathcal{P}=\left\{\mathbf{p}_{1}, \ldots, \mathbf{p}_{n}\right\}$ of $n$ points in $\mathbb{R}^{d}$ together with a set $\mathcal{E}$ of $m$ links, with $\mathcal{E} \subseteq\{(i, j), i \neq j, i, j \in\{1,2, \ldots, n\}\}$.

A point formation uniquely determines a directed graph $\mathcal{G}=(\mathcal{V}, \mathcal{E})$ with vertex set $\mathcal{V}=\{1,2, \ldots, n\}$ and edge set $\mathcal{E}$, together with a measurement function $\mathbf{u}: \mathcal{E} \rightarrow \mathbb{S}^{d-1}$ whose value at $(i, j) \in \mathcal{E}$ is the direction of $\mathbf{p}_{j}-\mathbf{p}_{i}$, which is defined in Equation (1). We assume here that the points in $\mathcal{F}_{\mathbf{p}}$ are distinct, i.e. $\mathbf{p}_{i} \neq \mathbf{p}_{j}$ for all $i \neq j$, so that Equation (1) is well defined. We use the notation $\mathbf{p} \in \mathbb{R}^{d n}$ to denote the stack of the coordinates of the points in $\mathcal{F}_{\mathbf{p}}$, that is $\mathbf{p}=$ $\left[\begin{array}{llll}\mathbf{p}_{1}^{\top} & \mathbf{p}_{2}^{\top} & \ldots & \mathbf{p}_{n}^{\top}\end{array}\right]^{\top}$.

A point formation represents a configuration of $n$ nodes in $d$-space. Specifically, the points $\mathbf{p}_{i}$ represent a solution to bearing-only localization with $\mathbf{u}_{i j}$ known, and the set $\mathcal{E}$ corresponds to node pairs for which the direction can be measured, which define constraints between specific nodes.

Definition 2. Two point formations $\mathcal{F}_{\mathbf{p}}$ and $\mathcal{F}_{\mathbf{q}}$ on the same graph $\mathcal{G}=(\mathcal{V}, \mathcal{E})$ are called parallel point formations (or parallel drawings) if $\mathbf{p}_{j}-\mathbf{p}_{i}$ is parallel to $\mathbf{q}_{j}-\mathbf{q}_{i}$ for all $(i, j) \in \mathcal{E}$.

Note that $\mathbf{p}_{j}-\mathbf{p}_{i}$ is parallel to $\mathbf{q}_{j}-\mathbf{q}_{i}$ if and only if there exists a scale $s_{i j} \in \mathbb{R}$ such that

$$
\mathbf{q}_{j}-\mathbf{q}_{i}=s_{i j}\left(\mathbf{p}_{j}-\mathbf{p}_{i}\right) \quad(i, j) \in \mathcal{E} .
$$

similarly to Equation (2).

The parallelism constraint for the edge $(i, j) \in \mathcal{E}$ can be expressed in an equivalent form which does not involve the unknown scale $s_{i j}$. Let us start with the $d=2$ case. Using the operator $(\cdot)^{\perp}$ for turning a plane vector by $\pi / 2$ counterclockwise, such constraint can be written as

$$
\left(\mathbf{p}_{j}-\mathbf{p}_{i}\right)^{\perp} \cdot\left(\mathbf{q}_{j}-\mathbf{q}_{i}\right)=0 \quad(i, j) \in \mathcal{E} .
$$

Thus, given a point formation $\mathcal{F}_{\mathbf{p}}$ in 2-space, a parallel drawing $\mathcal{F}_{\mathbf{q}}$ solves a homogeneous equation for each edge in $\mathcal{E}$, resulting in a linear system of $m$ equations in $2 n$ unknowns. In the $d \geqslant 3$ case, Equation (6) generalizes to

$$
\begin{array}{cc}
\left(\mathbf{p}_{j}-\mathbf{p}_{i}\right)_{N_{1}}^{\top}\left(\mathbf{q}_{j}-\mathbf{q}_{i}\right)=0 & (i, j) \in \mathcal{E} \\
\left(\mathbf{p}_{j}-\mathbf{p}_{i}\right)_{N_{2}}^{\top}\left(\mathbf{q}_{j}-\mathbf{q}_{i}\right)=0 & (i, j) \in \mathcal{E} \\
\cdots & \\
\left(\mathbf{p}_{j}-\mathbf{p}_{i}\right)_{N_{d-1}}^{\top}\left(\mathbf{q}_{j}-\mathbf{q}_{i}\right)=0 & (i, j) \in \mathcal{E}
\end{array}
$$

where $\left(\mathbf{p}_{j}-\mathbf{p}_{i}\right)_{N_{k}}$ for $k=1, \ldots, d-1$ are (linearly independent) vectors that span the subspace orthogonal to $\mathbf{p}_{j}-\mathbf{p}_{i}$. The equations in (7) are called the direction constraints (or normal constraints). Thus, given a point formation $\mathcal{F}_{\mathrm{p}}$, all parallel drawings solve $d-1$ homogeneous equations for each edge in $\mathcal{E}$. If we collect such equations for all the edges we get a system of $m(d-1)$ equations in $d n$ unknowns

$$
R_{\mathcal{F}_{\mathbf{p}}} \mathbf{q}=\mathbf{0}
$$

where $\mathbf{q}=\left[\begin{array}{llll}\mathbf{q}_{1}^{\top} & \mathbf{q}_{2}^{\top} & \ldots & \mathbf{q}_{n}^{\top}\end{array}\right]^{\top} \in \mathbb{R}^{d n}$ and $R_{\mathcal{F}_{\mathbf{p}}} \in \mathbb{R}^{m(d-1) \times d n}$ is called the parallel rigidity matrix.

Given a point formation $\mathcal{F}_{\mathbf{p}}$, trivially parallel point formations are translations and dilations of $\mathcal{F}_{\mathrm{p}}$ (including the parallel point formation in which all the points are coincident). Note that also a negative scaling of $\mathcal{F}_{\mathbf{p}}$ is considered trivially parallel to the original point formation. All the others (if they exist) are non trivial. Figure 2 shows an example: Figure 2a represents a point formation in $\mathbb{R}^{2}$; Figures $2 b$ and $2 \mathrm{c}$ are dilations of the point formation in Figure $2 \mathrm{a}$ (in particular, the former is an expansion and the latter is a contraction); Figure $2 \mathrm{~d}$ shows a translation of the point formation in Figure 2a; Figure 2e reports a non-trivially parallel point formation, since it can not be obtained from the point formation in Figure 2a by translation or dilation, although all the corresponding edges are parallel to each other.

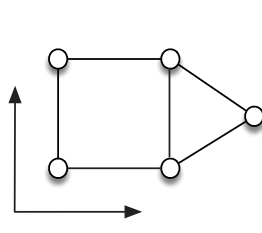

(a)

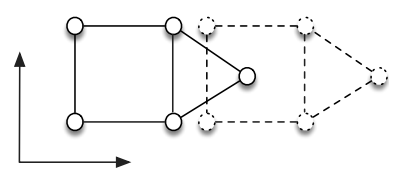

(d)

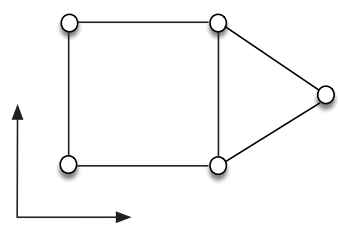

(b)

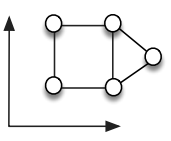

(c)

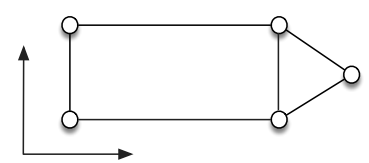

(e)

Figure 2: Parallel point formations.

Definition 3. A point formation $\mathcal{F}_{\mathrm{p}}$ is called parallel rigid (or tight) in $d$-space if all parallel point formations are trivially parallel. Otherwise it is called flexible (or loose).

According to Definition 3, the point formation in Figure 2a is flexible in $\mathbb{R}^{2}$ since it admits a non-trivial parallel drawing, whereas the point formation in Figure 3, which is obtained from the formation in Figure 2a by adding an extra link, is parallel rigid. Note that parallel rigidity is a 
property of the point formation, i.e. it depends both on the graph and on the coordinates of the points in $\mathbb{R}^{d}$.

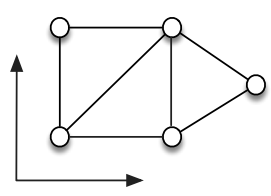

Figure 3: Parallel rigid point formation.

Note that trivially parallel point formations span a $(d+1)$-dimensional subspace of the null-space of $R_{\mathcal{F}_{\mathrm{p}}}$, corresponding to translations along each of $d$ axes and one scaling, thus $\operatorname{rank}\left(R_{\mathcal{F}_{\mathbf{p}}}\right) \leqslant d n-(d+1)$. Since the set of parallel drawings of a point formation $\mathcal{F}_{\mathrm{p}}$ coincides with the solution set of Equation 8, we obtain the following algebraic characterization of parallel rigidity.

Theorem 1 ( [16]). Suppose that a point formation $\mathcal{F}_{\mathbf{p}}$ contains at least $d+1$ points which are not contained in any proper hyperplane of $\mathbb{R}^{d} . \mathcal{F}_{\mathbf{p}}$ is parallel rigid in $d$-space if and only if

$$
\operatorname{dim}\left(\operatorname{ker}\left(R_{\mathcal{F}_{\mathbf{p}}}\right)\right)=d+1,
$$

or, equivalently, if and only if

$$
\operatorname{rank}\left(R_{\mathcal{F}_{\mathbf{p}}}\right)=d n-(d+1) .
$$

Note that the assumption in Theorem 1 guarantees that there are always $d+1$ independent solutions to (8). Otherwise, parallel rigidity can be checked in lower dimensions, namely in $(d-1)$-space if $\mathcal{F}_{\mathbf{p}}$ contains $d$ points, and so on. In the remainder of this section we suppose that such an assumption is satisfied.

Remark 1. We observe that a point formation $\mathcal{F}_{\mathbf{p}}=(\mathbf{p}, \mathcal{E})$ is parallel rigid if and only if $\mathcal{F}_{\mathbf{p}}^{\prime}=\left(\mathbf{p}, \mathcal{E}^{\prime}\right)$ is parallel rigid, where $\mathcal{E}^{\prime}$ is obtained from $\mathcal{E}$ by reversing the orientation of some edges, i.e. the endpoints are the same but the tail is replaced with the head and vice versa. Indeed, substituting $(i, j)$ with $(j, i)$ in Equation (7) results in an equivalent system. Thus the rigidity of a point formation is independent of the particular orientation of the edges, but it depends only on the underlying undirected graph. This is in agreement with Laman's condition (Theorem 3) which depends only on the number of edges/vertices of certain subgraphs of $\mathcal{G}$, but not on the orientation of the edges. For this reason, the figures in this paper represent undirected graphs. Note that in other related problems, e.g. formation control, the orientation of the edges is relevant, as explained by the notion of persistence [27], [28].

\subsubsection{Alternative Formulations of the Direction Constraints}

We observe that the directions constraints can be expressed in alternative forms which are equivalent to Equation (7). Let us start with the $d=3$ case. The vectors $\mathbf{p}_{j}-\mathbf{p}_{i}$ and $\mathbf{q}_{j}-\mathbf{q}_{i}$ are parallel if and only if their cross-product is zero, namely

$$
\left(\mathbf{p}_{j}-\mathbf{p}_{i}\right) \times\left(\mathbf{q}_{j}-\mathbf{q}_{i}\right)=\mathbf{0} \quad(i, j) \in \mathcal{E}
$$

or, equivalently,

$$
\left[\mathbf{p}_{j}-\mathbf{p}_{i}\right]_{\times}\left(\mathbf{q}_{j}-\mathbf{q}_{i}\right)=\mathbf{0} \quad(i, j) \in \mathcal{E}
$$

where $[\mathbf{a}]_{\times}$denotes the skew-symmetric matrix associated with the cross-product with $\mathbf{a}=\left[\begin{array}{lll}a_{1} & a_{2} & a_{3}\end{array}\right]^{\top}$, namely

$$
[\mathbf{a}]_{\times}=\left[\begin{array}{ccc}
0 & -a_{3} & a_{2} \\
a_{3} & 0 & -a_{1} \\
-a_{2} & a_{1} & 0
\end{array}\right] .
$$

Equation (12) gives rise to 3 homogeneous equations for each edge in $\mathcal{E}$, where only two of them are linearly independent. This formulation is used (e.g.) in [20], [21].

In the general case, the property that $\mathbf{p}_{j}-\mathbf{p}_{i}$ and $\mathbf{q}_{j}-\mathbf{q}_{i}$ are parallel can be expressed as a rank constraint, namely

$$
\operatorname{rank}\left(\left[\mathbf{p}_{j}-\mathbf{p}_{i}, \mathbf{q}_{j}-\mathbf{q}_{i}\right]\right)=1 \quad(i, j) \in \mathcal{E}
$$

which is equivalent to impose that all order-two minors are zero. The number of such minors is $d(d-1) / 2$, which give rise to $d(d-1) / 2$ homogeneous equations for each edge in $\mathcal{E}$, where only $d-1$ of them are linearly independent. Such equations can be expressed in matrix form as in Equation (12), where $[\mathbf{a}]_{\times}$now denotes a $d(d-1) / 2 \times d$ matrix composed of $d-1$ blocks arranged by rows, as explained in [29]. The $i$-th block has $d-i$ rows and $d$ columns

$$
A_{i}=\left[\begin{array}{ccccccc}
\mathbf{0}_{1 \times(i-1)} & -a_{i+1} & a_{i} & 0 & 0 & \ldots & 0 \\
\mathbf{0}_{1 \times(i-1)} & -a_{i+2} & 0 & a_{i} & 0 & \ldots & 0 \\
\mathbf{0}_{1 \times(i-1)} & -a_{i+3} & 0 & 0 & a_{i} & \ldots & 0 \\
\ldots & & & & & & \ldots \\
\mathbf{0}_{1 \times(i-1)} & -a_{d} & 0 & 0 & 0 & \ldots & a_{i}
\end{array}\right]
$$

and

$$
[\mathbf{a}]_{\times}=\left[\begin{array}{c}
A_{1} \\
\ldots \\
A_{d-1}
\end{array}\right] .
$$

It can be checked that Equation (16) reduces to (13) if $d=3$.

If we collect the equations in (12) for all the edges in $\mathcal{E}$, we get a system of $m d(d-1) / 2$ homogeneous equations in $d n$ unknowns. Let $S$ denote the $m d(d-1) / 2 \times m d$ blockdiagonal matrix with blocks $\left[\mathbf{p}_{j}-\mathbf{p}_{i}\right]_{\times}$along the diagonal, namely

$$
S=\operatorname{blockdiag}\left(\left\{\left[\mathbf{p}_{j}-\mathbf{p}_{i}\right]_{\times}\right\}_{(i, j) \in \mathcal{E}}\right)
$$

and let $Q=\left[\mathbf{q}_{1} \ldots \mathbf{q}_{n}\right]$. Using this notation, the equations in (12) can be expressed in a compact matrix form as

$$
S \operatorname{vec}(Q B)=\mathbf{0}
$$

which, using formula (90), rewrites

$$
S\left(B^{\top} \otimes I_{d}\right) \mathbf{q}=\mathbf{0}
$$

where $\mathbf{q}=\operatorname{vec}(Q), B$ is the incidence matrix and $\otimes$ is the Kronecker product. The linear system in (19) is equivalent to (8), thus we can check the rank of $S\left(B^{\top} \otimes I_{d}\right)$ instead of the rank of the parallel rigidity matrix to establish the rigidity of a point formation in $d$-space.

A different formulation is used in [9], [19], which is based on the observation that $\mathbf{p}_{j}-\mathbf{p}_{i}$ is parallel to $\mathbf{q}_{j}-\mathbf{q}_{i}$ if and only if the components of $\mathbf{q}_{j}-\mathbf{q}_{i}$ orthogonal to $\mathbf{p}_{j}-\mathbf{p}_{i}$ are zero, namely

$$
\Gamma_{\mathbf{p}_{j}-\mathbf{p}_{i}}\left(\mathbf{q}_{j}-\mathbf{q}_{i}\right)=\mathbf{0} \quad(i, j) \in \mathcal{E}
$$


where $\Gamma_{\mathbf{a}} \in \mathbb{R}^{d \times d}$ denotes the orthogonal projection matrix which geometrically projects any vector onto the orthogonal compliment of $\mathbf{a} \in \mathbb{R}^{d}$, namely

$$
\Gamma_{\mathbf{a}}=I_{d}-\frac{\mathbf{a} \mathbf{a}^{\top}}{\|\mathbf{a}\|^{2}} .
$$

Thus $d$ homogeneous equations for each edge in $\mathcal{E}$ are obtained, where only $d-1$ are linearly independent. Such equations can be collected for all the edges as in Equation (19), resulting in

$$
G\left(B^{\top} \otimes I_{d}\right) \mathbf{q}=\mathbf{0}
$$

where

$$
G=\operatorname{blockdiag}\left(\left\{\Gamma_{\mathbf{p}_{j}-\mathbf{p}_{i}}\right\}_{(i, j) \in \mathcal{E}}\right) .
$$

Equation (22) is a system of $d m$ homogeneous equations in $d n$ unknowns which is equivalent to (8), thus the rank of $G\left(B^{\top} \otimes I_{d}\right)$ can be computed to check the rigidity of a point formation.

\subsubsection{The Bearing Laplacian Matrix}

In practice, a parallel point formation $\mathbf{q}$ can be recovered by solving the normal equations associated with (22), namely

$$
\underbrace{\left(G\left(B^{\top} \otimes I_{d}\right)\right)^{\top}\left(G\left(B^{\top} \otimes I_{d}\right)\right)}_{H} \mathbf{q}=\mathbf{0} .
$$

Note that $H$ is symmetric and positive semidefinite. By computation it can be verified that the projection matrix $\Gamma_{\mathbf{a}}$ defined in (21) satisfies $\Gamma_{\mathbf{a}}^{\top}=\Gamma_{\mathbf{a}}=\Gamma_{\mathbf{a}}^{2}$ for all $\mathbf{a} \in \mathbb{R}^{d}$, thus $G^{\top} G=G$. Using this property and Equation (87) we get

$$
H=\left(B \otimes I_{d}\right) G\left(B^{\top} \otimes I_{d}\right)
$$

which, after some rewriting, can be expressed as

$$
\begin{aligned}
H= & {\left[\begin{array}{cccc}
\sum_{j} \Gamma^{1 j} & & & \\
& \sum_{j} \Gamma^{2 j} & & \\
& & \ddots & \\
& & & \sum_{j} \Gamma^{n j}
\end{array}\right]+} \\
& -\left[\begin{array}{cccc}
0 & \Gamma^{12} & \ldots & \Gamma^{1 n} \\
\Gamma^{21} & 0 & \ldots & \Gamma^{2 n} \\
\vdots & & \ddots & \vdots \\
\Gamma^{n 1} & \Gamma^{n 2} & \ldots & 0
\end{array}\right]
\end{aligned}
$$

where, for simplicity of notation, $\Gamma^{i j}=\Gamma_{\mathbf{p}_{j}-\mathbf{p}_{i}}$. The matrix $H$ is called the bearing Laplacian in [9] since it resembles the Laplacian matrix of a weighted graph (see Appendix A.2). Note that Equation (26) is the same as the matrix used in [30]. Since $\operatorname{rank}(H)=\operatorname{rank}\left(G\left(B^{\mathrm{\top}} \otimes I_{d}\right)\right)$, the rank of the bearing Laplacian matrix can be computed in order to check the rigidity of a point formation in d-space, as done in [9].

\subsubsection{Global Rigidity}

We introduce the concept of global parallel rigidity which turns out to be equivalent to parallel rigidity. In the case of distance-based localization, instead, the conditions for global rigidity are stronger than those for rigidity [26].

In the same way as a point formation uniquely determines a graph and a measurement function, it also determines a localization problem where the input are such graph and directions. By construction, the point formation solves this problem, thus the question is whether it is the unique (up to translation and scale) solution (or there exist other nontrivially parallel solutions). If the answer is positive, then the configuration is called globally parallel rigid. In other words, a point formation is globally parallel rigid if it is the unique solution to the associated localization problem.

Definition 4. A point formation $\mathcal{F}_{\mathbf{p}}$ is called globally parallel rigid in $d$-space if it is exactly determined (up to translation and scale) by its graph and measurement function.

Proposition 1 ( [17]). A point formation $\mathcal{F}_{\mathbf{p}}$ is parallel rigid if and only if it is globally parallel rigid.

Proof. We follow the reasoning reported in [31]. Let us consider the measurement function which associates a point formation $\mathcal{F}_{\mathbf{p}}$ with $\Gamma_{\mathbf{p}_{j}-\mathbf{p}_{i}}$ for all $(i, j) \in \mathcal{E}$, where the orthogonal projection matrix $\Gamma$ is defined in (21). Note that the knowledge of $\Gamma_{\mathbf{p}_{j}-\mathbf{p}_{i}}$ is equivalent to the knowledge of $\pm \mathbf{u}_{i j}$ where the sign is undetermined, with $\mathbf{u}_{i j}$ defined in (1). Let us define a map $\mathcal{M}$ that assigns each point formation to its graph and measurement function, namely

$$
\mathcal{M}: \mathcal{F}_{\mathbf{p}} \mapsto(\mathcal{G}, \Gamma)
$$

More precisely, since the property of being trivially parallel point formation is an equivalence relation, we can define the above map on the quotient space induced by such relation. Note that $\mathcal{F}_{\mathbf{p}}$ is globally parallel rigid if and only if $\mathcal{M}$ is injective at $\mathcal{F}_{\mathbf{p}}$. It is clear that $\mathcal{M}$ is injective at $\mathcal{F}_{\mathbf{p}}$ if and only if $\mathcal{F}_{\mathbf{p}}$ admits only trivially parallel point formations, since parallel point formations have the same measurement function and vice-versa.

Given the interpretation of global rigidity in terms of bearing-based localization, Proposition 1 implies that parallel rigidity is equivalent to the unique localizability of a sensor network in d-space. Accordingly, the equations arising from the definition of parallel rigidity can be exploited to address the localization problem via a node-based approach. In order to facilitate the implementation, we refer here to the formulations reported in Section 2.1.1, which are equivalent to Equation (8).

Given a set of directions $\left\{\mathbf{u}_{i j}\right\}$ for $(i, j) \in \mathcal{E}$, the localization problem can be addressed by solving the following homogeneous system of equations with respect to the unknown $\mathbf{p} \in \mathbb{R}^{d n}$

$$
S\left(B^{\top} \otimes I_{d}\right) \mathbf{p}=\mathbf{0}
$$

where $S=\operatorname{blockdiag}\left(\left\{\left[\mathbf{u}_{i j}\right]_{\times}\right\}_{(i, j) \in \mathcal{E}}\right)$. This formulation is exploited in [32], [33] in the context of structure from motion. The above equation has the same structure as (19): in the first case, we are looking for a point formation which represents the solution to bearing-based localization - whose edges are parallel to a given set of directions; in the second case, we are looking for point formations whose edges are parallel to those of a given configuration. Note that $\left\|\mathbf{u}_{i j}\right\|\left[\mathbf{u}_{i j}\right]_{\times}=\left[\mathbf{p}_{j}-\mathbf{p}_{i}\right]_{\times}$, thus the matrices in Equations (19) and (28) coincide up to a left-multiplication by a diagonal matrix of scales. As a consequence, the rank of the matrix in (28) - instead of (19) - can be computed to establish the rigidity of $\mathcal{F}_{\mathbf{p}}$ in d-space, or, equivalently, the uniqueness of the solution to bearing-based localization, in agreement with Proposition 1. 
Remark 2. Equation (28) can also be derived by multiplying (4) by the block-diagonal matrix $S$

$$
S\left(B^{\boldsymbol{\top}} \otimes I_{d}\right) \mathbf{p}=S\left(I_{m} \odot U\right) \boldsymbol{\alpha} .
$$

Note that, by construction, the right-side vanishes, yielding Equation (28), which does not involve the edge scales. Alternatively, node locations and edge scales can be computed simultaneously from Equation (4), as done in [34], [35], [36].

A different formulation for bearing-based localization is used in [9], [19], [30], [37] where the following homogeneous system of equations is considered

$$
G\left(B^{\top} \otimes I_{d}\right) \mathbf{p}=\mathbf{0}
$$

with $G=$ blockdiag $\left(\left\{\Gamma_{\mathbf{u}_{i j}}\right\}_{(i, j) \in \mathcal{E}}\right)$, which resembles Equation (22) in the same way as (28) resembles Equation (19).

Remark 3. In the $d=3$ case the bearing Laplacian associated with Equation (30) can be equivalently expressed as

$$
H=\left(B \otimes I_{d}\right) G\left(B^{\top} \otimes I_{d}\right)=\left(B \otimes I_{d}\right) S^{\top} S\left(B^{\top} \otimes I_{d}\right)
$$

since $[\mathbf{a}]_{\times}[\mathbf{b}]_{\times}=\mathbf{b a}^{\top}-\mathbf{a b}^{\top} I_{3}$ for all $\mathbf{a}, \mathbf{b} \in \mathbb{R}^{3}$, which implies that $\left[\mathbf{u}_{i j}\right]_{\times}^{\top}\left[\mathbf{u}_{i j}\right]_{\times}=I_{3}-\mathbf{u}_{i j} \mathbf{u}_{i j}^{\top}=\Gamma_{\mathbf{u}_{i j}}$ (recall that $\left\|\mathbf{u}_{i j}\right\|=1$ by construction). Note that the matrix in Equation (31) is the coefficient matrix of the normal equations associated with (28). This implies that (in the presence of noise) the least-squares solution associated with (28) coincides with the spectral solution proposed in [30], as observed also in [21].

\subsection{Generic Rigidity}

According to the above results, given a point formation $\mathcal{F}_{\mathbf{p}}$, it can be established (by checking the rank of the parallel rigidity matrix) if it is uniquely determined by its graph and directions. If it is not so, the cause can be the structure of the graph or the actual coordinates of the points, i.e., there can be algebraic dependencies among the coordinates that make the rank drop. How can we predict the rigidity of the problem based on the structure of the graph (and dimension d) only? This issue is addressed in the present section, where the concept of generic parallel rigidity is introduced. This property does not depend on the specific coordinates of a point formation, but it predicts the rigidity of almost all the point formations from the nodes and their incidences, i.e., from the underlying graph.

Definition 5. A set $\mathcal{A}=\left\{\alpha_{1}, \ldots, \alpha_{k}\right\}$ of distinct real numbers is called algebraically dependent if there exists a non-zero polynomial $h$ with integer coefficients such that $h\left(\alpha_{1}, \ldots, \alpha_{k}\right)=0$. Otherwise it is called generic.

A set $\mathcal{P}=\left\{\mathbf{p}_{1}, \ldots, \mathbf{p}_{n}\right\}$ of points in $\mathbb{R}^{d}$ is called generic if its $d n$ coordinates are generic. Note that if the set contains less than $d+1$ points, then there are always algebraic dependencies among the coordinates, since the points are contained in a hyperplane of $\mathbb{R}^{d}$. In such a situation the generic property is checked in lower dimensions (namely $d-1$ if there are $d$ points, and so on). It can be shown that the set of generic $\mathcal{P}$ s forms an open dense subset of $\mathbb{R}^{d n}$ [12]. Note that, with reference to the structure-from-motion application, a camera that is moving along a straight line is not considered generic by this theory (if the points in the scene are not considered).

Definition 6. A graph $\mathcal{G}=(\mathcal{V}, \mathcal{E})$ is called generically parallel rigid in $d$-space if $\mathcal{F}_{\mathbf{p}}=(\mathcal{P}, \mathcal{E})$ is parallel rigid for a generic $\mathcal{P}$. Otherwise it is called generically flexible.

Due to Theorem 1, we can equivalently say that a graph $\mathcal{G}=(\mathcal{V}, \mathcal{E})$ is generically parallel rigid if and only if $\operatorname{rank}\left(R_{\mathcal{F}_{\mathbf{p}}}\right)=d n-(d+1)$, where $R_{\mathcal{F}_{\mathbf{p}}}$ is constructed using a generic point formation $\mathcal{F}_{\mathrm{p}}$. It can be shown that $\operatorname{rank}\left(R_{\mathcal{F}_{\mathbf{p}}}\right)$ is independent of the coordinates of the chosen point formation (assuming that it is generic) and it depends only on the underlying graph and dimension $d$ [16], hence Definition 6 is well-posed. Due to Proposition 1, we can equivalently say that a graph $\mathcal{G}=(\mathcal{V}, \mathcal{E})$ is generically parallel rigid if $\mathcal{F}_{\mathbf{p}}=(\mathcal{P}, \mathcal{E})$ is globally parallel rigid for a generic $\mathcal{P}$.

Figure 4a shows a generically parallel rigid graph ${ }^{1}$. It is easy to see that, given a point formation with generic coordinates, all the possible transformations that keep the corresponding edges parallel are translations and dilations, thus the underlying graph is parallel rigid in $d$-space. Note that the rigidity of $\mathcal{G}$ does not imply that all the point formations defined on $\mathcal{G}$ are parallel rigid. If there are algebraic dependencies among the coordinates (e.g. all the nodes lie on a common line) then the rank of the parallel rigidity matrix drop, resulting in a flexible point formation. The rigidity of $\mathcal{G}$ implies that all the generic point formations are parallel rigid, and hence, since generic formations are dense in $\mathbb{R}^{d n}$, it implies that almost all the formations defined on $\mathcal{G}$ are parallel rigid in $d$-space. Figure $4 \mathrm{~b}$ shows a generically flexible graph $\mathcal{G}=(\mathcal{V}, \mathcal{E})$. It is easy to see that, given a point formation with generic coordinates, independent scaling of the edges in the subgraphs $\mathcal{G}_{1}=\left(\mathcal{V}_{1}, \mathcal{E}_{1}\right)=(\{1,2,3\},\{(1,2),(2,3),(3,1)\})$ and $\mathcal{G}_{2}=\left(\mathcal{V}_{2}, \mathcal{E}_{2}\right)=(\{3,4,5\},\{(3,4),(4,5),(5,3)\})$ produces non-trivially parallel point formations, thus the underlying graph is flexible in $d$-space.

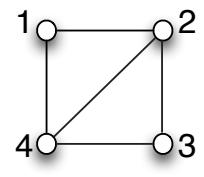

(a)

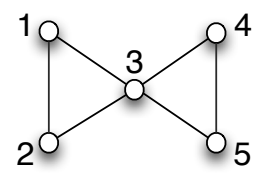

(b)
Figure 4: Left: generically parallel rigid graph. Right: generically flexible graph.

Note that, if we choose a graph and vary the position of the nodes in $d$-space, the dimension of the solution space of Equation (8) changes. However, a minimum dimension occurs and it clearly depends only on the underlying graph. It can be shown that such minimum is attained when the coordinates of the nodes are generic [16], which is equivalent to say that generic point formations maximize the

1. Note that Figures 2 and 3 represent 2-dimensional point formations, i.e. specific coordinates of nodes in 2-space, whereas Figure 4 and all subsequent figures represent graphs, i.e. only the links matter and the embedding in the plane is merely accidental. To make this difference evident a $2 \mathrm{D}$ reference frame is drawn where appropriate. 
rank of the parallel rigidity matrix. Recall that $\operatorname{rank}\left(R_{\mathcal{F}_{\mathrm{p}}}\right)$ is constant for any generic $\mathcal{F}_{\mathbf{p}}$. Thus we get the following algebraic characterization of generic parallel rigidity.

Theorem 2 ( [16]). A graph $\mathcal{G}=(\mathcal{V}, \mathcal{E})$ is generically parallel rigid in $d$-space if and only if

$$
\max _{p \in \mathbb{R}^{n d}} \operatorname{rank}\left(R_{\mathcal{F}_{\mathbf{p}}}\right)=d n-(d+1) .
$$

Theorem 2 gives rise to a randomized test for checking generic parallel rigidity [19], where a parallel rigidity matrix is built from a point formation randomly sampled from i.i.d. Gaussian distribution. This test correctly establishes the generic rigidity of $\mathcal{G}$ with probability 1 with a time complexity of $O(m)$, with $m=|\mathcal{E}|$.

Note that, if $\mathcal{G}$ is generically parallel rigid, then adding edges between existing nodes keeps the graph rigid, since it corresponds to include dependent equations in (8). If removing an edge results in a flexible graph, then $\mathcal{G}=(\mathcal{V}, \mathcal{E})$ is called minimally parallel rigid in $d$-space, i.e. it is generically parallel rigid with minimum number of constraints. See [15] for techniques to generate minimally rigid graphs in 2-space and in 3-space.

We now list some combinatorial characterizations of generic parallel rigidity.

Theorem 3 (Laman's condition [11]). A graph $\mathcal{G}=(\mathcal{V}, \mathcal{E})$ is generically parallel rigid in $d$-space if and only if there exists a subset $\mathcal{E}^{\prime} \subseteq(d-1) \mathcal{E}$, where $(d-1) \mathcal{E}$ denotes the set consisting of $d-1$ copies of the edges in $\mathcal{E}$, such that the following conditions are satisfied:

1) $\left|\mathcal{E}^{\prime}\right|=d n-(d+1)$;

2) $\forall \mathcal{E}^{\prime \prime} \subseteq \mathcal{E}^{\prime}, \mathcal{E}^{\prime \prime} \neq \varnothing:\left|\mathcal{E}^{\prime \prime}\right| \leqslant d\left|\mathcal{V}^{\prime \prime}\right|-(d+1)$, where $\mathcal{V}^{\prime \prime}$ denotes the set of vertices that are endpoints of the edges in $\mathcal{E}^{\prime \prime}$.

Corollary 1 ( [10]). A graph $\mathcal{G}=(\mathcal{V}, \mathcal{E})$ is generically parallel rigid in $d$-space if and only if for any partition $\left\{\mathcal{E}^{1}, \mathcal{E}^{2}, \ldots, \mathcal{E}^{h}\right\}$ of $\mathcal{E}$ it holds

$$
\sum_{i=1}^{h}\left(d\left|\mathcal{V}^{i}\right|-(d+1)\right) \geqslant d n-(d+1) .
$$

The conditions in Theorem 3 translate into combinatorial algorithms for testing generic parallel rigidity, e.g. methods based on the pebble game [38] with a time complexity of $O\left(n^{2}\right)$. Note that if $\mathcal{E}$ satisfies $(d-1)|\mathcal{E}|<d n-(d+1)$ then, even if we take $\mathcal{E}^{\prime}=(d-1) \mathcal{E}$, Condition 1 in Theorem 3 will not be fulfilled. Thus we have the following necessary condition for a graph $\mathcal{G}$ to be generically parallel rigid

$$
(d-1) m \geqslant d n-(d+1)
$$

which essentially states that $\mathcal{G}$ needs to have a sufficient number of edges.

Let us consider the examples provided in Figure 4. The graph in Figure $4 \mathrm{a}$ is generically parallel rigid in 2-space, since $\mathcal{E}^{\prime}=\mathcal{E}$ satisfies the conditions in Theorem 3, whereas the graph in Figure $4 \mathrm{~b}$ is generically flexible in 2 -space, since the necessary condition (34) is not satisfied. Note that in the $d=2$ case the set $\mathcal{E}^{\prime}$ satisfying the conditions in Theorem 3 (if it exists) is simply a subset of the edge set $\mathcal{E}$. We now consider the $d=3$ case. As for Figure $4 \mathrm{a}$, it is easy to see that $\mathcal{E}^{\prime}=\mathcal{E} \cup\{(1,2),(2,4),(4,1)\} \subseteq 2 \mathcal{E}$ satisfies the conditions in Theorem 3. As for Figure $4 \mathrm{~b}$, since $|2 \mathcal{E}|=12$, there exists a set $\mathcal{E}^{\prime}$ satisfying Condition 1, i.e. $\left|\mathcal{E}^{\prime}\right|=11$, only if $\mathcal{E}^{\prime}=$ $2 \mathcal{E} \backslash\{e\}$ for some $e \in \mathcal{E}$. However, in this case $\mathcal{E}^{\prime}$ has a subset $\mathcal{E}^{\prime \prime}$ consisting of two copies of each of the three edges in a triangle graph, which violates Condition 2.

The following result derives from the equivalence between the count in Theorem 3 and a $(d+1) T d$ decomposition of $\mathcal{E}^{\prime}$ [39], i.e. a decomposition of $\mathcal{E}^{\prime}$ into $d+1$ edgedisjoint trees where each vertex is contained in $d$ trees. See Appendix A for the definition of tree.

Theorem 4 ( [11]). A graph $\mathcal{G}=(\mathcal{V}, \mathcal{E})$ is generically parallel rigid in $d$-space if and only if there exists a subset $\mathcal{E}^{\prime} \subseteq(d-1) \mathcal{E}$ such that $\mathcal{E}^{\prime}$ can be decomposed into $d+1$ edge-disjoint trees, where each vertex is contained in exactly $d$ trees, and for any subgraph $\mathcal{E}^{\prime \prime} \subseteq \mathcal{E}^{\prime}, \mathcal{E}^{\prime \prime} \neq \varnothing$, the set of trees induced by $\mathcal{E}^{\prime \prime}$ has cardinality at least $d+1$.

Note that Theorems 3 and 4 do not involve the whole graph $\mathcal{G}=(\mathcal{V}, \mathcal{E})$, but the existence of a subset $\mathcal{E}^{\prime} \subseteq(d-1) \mathcal{E}$ with certain properties. As observed in [10], [11], such a subset corresponds to $d n-(d+1)$ linearly independent rows of the parallel rigidity matrix, whose existence is equivalent to $\operatorname{rank}\left(R_{\mathcal{F}_{\mathbf{p}}}\right)=d n-(d+1)$.

The following proposition, which exploits Laman's condition, establishes the relation between generic rigidity in $d$-space and in $(d+1)$-space. In particular, we get that all the graphs which are generically parallel rigid in $\mathbb{R}^{2}$ are also rigid in $\mathbb{R}^{d}$ for any $d \geqslant 3$.

Proposition 2. If a graph $\mathcal{G}=(\mathcal{V}, \mathcal{E})$ is generically parallel rigid in $d$-space, then it is generically parallel rigid in $(d+1)$-space.

Proof. Since $\mathcal{G}=(\mathcal{V}, \mathcal{E})$ is generically parallel rigid in $d$ space, by virtue of Theorem 3 , there exists a subset $\mathcal{E}^{\prime} \subseteq$ $(d-1) \mathcal{E}$ such that

$$
\begin{gathered}
\left|\mathcal{E}^{\prime}\right|=d n-(d+1) \\
\forall \mathcal{E}^{\prime \prime} \subseteq \mathcal{E}^{\prime}, \mathcal{E}^{\prime \prime} \neq \varnothing: \quad\left|\mathcal{E}^{\prime \prime}\right| \leqslant d\left|\mathcal{V}^{\prime \prime}\right|-(d+1) .
\end{gathered}
$$

Note that $\mathcal{G}$ is connected, otherwise a non-trivial parallel drawing can be found from independent scaling and/or translation of each connected component, which contradicts the assumption. In order to prove that $\mathcal{G}$ is generically parallel rigid in $(d+1)$-space, we have to find a set $\widetilde{\mathcal{E}}^{\prime} \subseteq d \mathcal{E}$ satisfying the following conditions

$$
\begin{gathered}
\left|\widetilde{\mathcal{E}}^{\prime}\right|=(d+1) n-(d+2) \\
\forall \widetilde{\mathcal{E}}^{\prime \prime} \subseteq \widetilde{\mathcal{E}}^{\prime}, \widetilde{\mathcal{E}}^{\prime \prime} \neq \varnothing:\left|\widetilde{\mathcal{E}}^{\prime \prime}\right| \leqslant(d+1)\left|\widetilde{\mathcal{V}}^{\prime \prime}\right|-(d+2) .
\end{gathered}
$$

Let us define $\widetilde{\mathcal{E}}^{\prime}:=\mathcal{E}^{\prime} \cup \mathcal{T}$, where $\mathcal{T}$ is any arbitrary spanning tree of $\mathcal{G}$, which is well defined since $\mathcal{G}$ is connected (see Appendix A). Note that $\mathcal{E}^{\prime}$ is contained in the set consisting of $d-1$ copies of the edges of $\mathcal{E}$, whereas $\mathcal{T}$ is contained in $\mathcal{E}$, thus their union is contained in the set consisting of $d$ copies of the edges of $\mathcal{E}$. Using Equation (35) and $|\mathcal{T}|=n-1$ we get $\left|\widetilde{\mathcal{E}}^{\prime}\right|=\left|\mathcal{E}^{\prime}\right|+|\mathcal{T}|=d n-(d+1)+(n-1)=(d+1) n-(d+2)$ and hence Equation (37) is satisfied. We now prove that Equation (38) holds. Let $\widetilde{\mathcal{E}}^{\prime \prime} \subseteq \widetilde{\mathcal{E}}^{\prime}$ with $\widetilde{\mathcal{E}}^{\prime \prime} \neq \varnothing$. We can write $\widetilde{\mathcal{E}}^{\prime \prime}=$ $\mathcal{E}^{\prime \prime} \cup \mathcal{T}^{\prime \prime}$ where $\mathcal{E}^{\prime \prime} \subseteq \mathcal{E}^{\prime}$ and $\mathcal{T}^{\prime \prime} \subseteq \mathcal{T}$. Note that $\mathcal{T}^{\prime \prime}$ is not necessarily a tree, but it will be a disjoint union of trees (i.e. 
a forest) in general, thus $\left|\mathcal{T}^{\prime \prime}\right|=n_{\mathcal{T}}{ }^{\prime \prime}-c c \leqslant n_{\mathcal{T}} \prime \prime-1$, where $c c$ denotes the number of connected components in $\mathcal{T}^{\prime \prime}$ and $n_{\mathcal{T}}$ " denotes the number of vertices that are endpoints of the edges in $\mathcal{T}^{\prime \prime}$. Combining this observation with Equation (36) we get $\left|\widetilde{\mathcal{E}}^{\prime \prime}\right|=\left|\mathcal{E}^{\prime \prime}\right|+\left|\mathcal{T}^{\prime \prime}\right| \leqslant d\left|\mathcal{V}^{\prime \prime}\right|-(d+1)+n_{\mathcal{T}}^{\prime \prime}-1$. Note that the number of vertices that are endpoints of the edges in $\mathcal{E}^{\prime \prime}$ and the number of vertices that are endpoints of the edges in $\mathcal{T}^{\prime \prime}$ are both dominated by the total number of vertices in $\widetilde{\mathcal{E}}^{\prime \prime}$, i.e. $\left|\mathcal{V}^{\prime \prime}\right| \leqslant\left|\widetilde{\mathcal{V}}^{\prime \prime}\right|$ and $n_{\mathcal{T}^{\prime \prime}} \leqslant\left|\widetilde{\mathcal{V}}^{\prime \prime}\right|$, hence we get Equation (38).

Note that the converse of Proposition 2 is not true. For instance, the graph associated with the point formation in Figure 2 is flexible in $\mathbb{R}^{2}$ and it is parallel rigid in $\mathbb{R}^{d}$ for any $d \geqslant 3$, as it can be checked easily.

\subsubsection{Maximal Rigid Components}

If a graph is not generically parallel rigid, then it can be decomposed into maximal rigid components. A rigid component of $\mathcal{G}=(\mathcal{V}, \mathcal{E})$ in $d$-space is a subgraph $\mathcal{G}^{\prime} \subseteq \mathcal{G}$ such that $\mathcal{G}^{\prime}$ is generically parallel rigid in $d$-space. Clearly, the union of rigid components sharing (at least) one edge is also rigid, since the edge in common fixes the position of two nodes and hence it determines the global scale and translation. A rigid component is called maximal if it is not a subset of any other rigid component. A maximal rigid component is called the largest maximal rigid component if it has the largest number of nodes.

Theorem 5 ( [21]). The set of all maximal rigid components of a graph $\mathcal{G}=(\mathcal{V}, \mathcal{E})$ in $d$-space induces a partition of the edge set $\mathcal{E}$.

For instance, the edge set of the (flexible) graph reported in Figure $4 \mathrm{~b}$ can be partitioned into two maximal rigid components, namely $\mathcal{E}^{1}=\{(1,2),(2,3),(3,1)\}$ and $\mathcal{E}^{2}=\{(3,4),(4,5),(5,3)\}$. Note that these components share one vertex.

Polynomial-time algorithms for finding maximal rigid components of flexible graphs are presented in [21], [22]. The authors of [21] analyze the null-space of the parallel rigidity matrix and cast the problem to identifying sets of parallel lines. A different approach is followed in [22] where rigid components are first identified among known rigid graphs of small size, and then they are grouped using a reduction to a maximum flow problem.

We briefly review here the algorithm introduced in [21]. Let $R_{\mathcal{F}_{\mathbf{p}}}$ be the parallel rigidity matrix associated with a generic point formation $\mathcal{F}_{\mathbf{p}}$ and let $N \in \mathbb{R}^{d n \times k}$ be a matrix whose columns span the null-space of $R_{\mathcal{F}_{\mathbf{p}}}$, where

$$
k=\operatorname{dim}\left(\operatorname{ker}\left(R_{\mathcal{F}_{\mathbf{p}}}\right)\right)>d+1 .
$$

Equivalently, the matrices in Equations (19) or (22) could be used in place of $R_{\mathcal{F}_{\mathbf{p}}}$, as explained in Section 2.1.1. The null-space matrix $N$ can be partitioned into blocks as

$$
N=\left[\begin{array}{c}
M_{1} \\
\cdots \\
M_{i} \\
\cdots \\
M_{n}
\end{array}\right]
$$

where $M_{i} \in \mathbb{R}^{d \times k}$ denotes the $i$-th set of $d$ rows of $N$ corresponding to node $i$.

Theorem 6 ( [21]). Let us fix a node $i$ and consider the modified null-space matrix

$$
N^{i}=\left[\begin{array}{c}
M_{1}-M_{i} \\
\ldots \\
0 \\
\cdots \\
M_{n}-M_{i}
\end{array}\right] \leftarrow i .
$$

Nodes $j$ and $h$ with $j \neq i$ and $h \neq i$ ) are part of a maximal rigid component with node $i$ if and only if the $d$ rows of $N^{i}$ corresponding to node $j$ are parallel to the corresponding rows for node $h$.

Theorem 6 implies that we can find the vertex sets (and hence the corresponding edge sets) of all maximal rigid components containing node $i$ by identifying sets of parallel rows in $N^{i}$, where, as suggested in [21], the cosine similarity can be used to check if two vectors are parallel. By repeating this procedure for each node, the flexible graph $\mathcal{G}$ is decomposed into maximal rigid components, with a total cost of $O\left(k n^{3}\right)$, as explained in [21].

\section{Edge-based Parallel Rigidity}

In this section we describe an equivalent formulation of parallel rigidity, which is called the edge-based formulation, since it reasons in terms of edge lengths rather than node positions. It provides a more intuitive way to look at rigidity, since the problem is expressed in terms of cycles in the graph. This formulation is based on some recent works [14], [20], [22].

\subsection{Rigidity of a Point Formation}

Let $\mathcal{F}_{\mathbf{p}}$ be a point formation in $d$-space and let $\alpha_{i j} \in \mathbb{R}^{+}$ denote the length of $\mathbf{p}_{j}-\mathbf{p}_{i}$ for $(i, j) \in \mathcal{E}$, namely

$$
\alpha_{i j}=\left\|\mathbf{p}_{j}-\mathbf{p}_{i}\right\| \text {. }
$$

The inter-nodal distances $\alpha_{i j}$ are called the epipolar scales in [14], with reference to the structure-from-motion application, and edge scales in [20]. Alternatively, given a point formation $\mathcal{F}_{\mathbf{p}}$ and its measurement function, i.e. its set of directions $\left\{\mathbf{u}_{i j}\right\}$, we can define the length of edge $(i, j)$ as the positive real number $\alpha_{i j}$ such that Equation (2) holds, i.e. $\mathbf{p}_{j}-\mathbf{p}_{i}=\alpha_{i j} \mathbf{u}_{i j}$. This general definition can also take into account the fact that a direction $\mathbf{u}_{i j}$ may be measured with the wrong sign, in which case the corresponding $\alpha_{i j}$ is negative in order to fulfill Equation (2).

We now show how parallel rigidity can be restated in terms of edge lengths.

Proposition 3 ( [20]). A point formation $\mathcal{F}_{\mathbf{p}}$ is parallel rigid if and only if for any parallel point formation $\mathcal{F}_{\mathbf{q}}$ it holds

$$
\mathbf{q}_{j}-\mathbf{q}_{i}=s\left(\mathbf{p}_{j}-\mathbf{p}_{i}\right) \quad \forall(i, j) \in \mathcal{E}
$$

assuming that $\mathcal{G}=(\mathcal{V}, \mathcal{E})$ is connected, where $s$ does not depend on the individual $(i, j)$ pair.

Proof. In one direction: if $\mathcal{F}_{\mathbf{p}}$ is parallel rigid then - by definition - any parallel drawing $\mathcal{F}_{\mathbf{q}}$ satisfies $\mathbf{q}_{i}=s \mathbf{p}_{i}+\mathbf{t}$ with 
$s \in \mathbb{R}$ and $\mathbf{t} \in \mathbb{R}^{d}$, and hence Equation (43) clearly holds. In the opposite direction: let $\mathcal{F}_{\mathbf{p}}$ be a point formation and let $\mathcal{F}_{\mathbf{q}}$ be a parallel drawing such that $\mathbf{q}_{j}-\mathbf{q}_{i}=s\left(\mathbf{p}_{j}-\mathbf{p}_{i}\right)$ for all $(i, j) \in \mathcal{E}$, or, equivalently, $\mathbf{q}_{j}-s \mathbf{p}_{j}=\mathbf{q}_{i}-s \mathbf{p}_{i}$. If the graph is connected, each node can be reached by any other node through a path, thus such relation is valid for all the nodes, i.e. $\mathbf{q}_{j}-s \mathbf{p}_{j}=\mathbf{q}_{i}-s \mathbf{p}_{i}=\cdots=\mathbf{q}_{k}-s \mathbf{p}_{k}=\mathbf{t}$, thus $\mathbf{q}_{i}=s \mathbf{p}_{i}+\mathbf{t}$ for all $i \in \mathcal{V}$, which means that $\mathcal{F}_{\mathbf{p}}$ is parallel rigid.

A set of edges satisfying Equation (43) is called an interdependent edge set in [20]. Note that, according to Equation (5), the parallelism of $\mathcal{F}_{\mathbf{p}}$ and $\mathcal{F}_{\mathbf{q}}$ rewrites $\mathbf{q}_{j}-\mathbf{q}_{i}=$ $s_{i j}\left(\mathbf{p}_{j}-\mathbf{p}_{i}\right)$ for some scales $s_{i j} \in \mathbb{R}$, while parallel rigidity - as expressed by Equation (43) - means that such scales are all equal, i.e. $s_{i j}=s \in \mathbb{R}$ for all $(i, j) \in \mathcal{E}$.

Proposition 4. A point formation $\mathcal{F}_{\mathbf{p}}$ is parallel rigid if and only if its lengths are exactly determined (up to a global scale) by its graph and measurement function, assuming that the underlying graph $\mathcal{G}=(\mathcal{V}, \mathcal{E})$ is connected.

Proof. In one direction: if $\mathcal{F}_{\mathbf{p}}$ is parallel rigid then, due to (43), any parallel drawing $\mathcal{F}_{\mathbf{q}}$ satisfies $\left\|\mathbf{q}_{j}-\mathbf{q}_{i}\right\|=$ $|s| \cdot|| \mathbf{p}_{j}-\mathbf{p}_{i} \|$ for all $(i, j) \in \mathcal{E}$, i.e. the lengths of $\mathcal{F}_{\mathbf{q}}$ coincide (up to a global scale) with those of $\mathcal{F}_{\mathbf{p}}$. Recall that parallel point formations have the same measurement function and vice-versa, hence we get the thesis. In the opposite direction: by definition, the lengths of $\mathcal{F}_{\mathbf{p}}$ satisfy (2), which is equivalent to Equation (4). If we assume that the graph is connected then $\operatorname{rank}(B)=n-1$ (see Appendix A) and hence, using (92), $\operatorname{rank}\left(B^{\top} \otimes I_{d}\right)=d n-d$, where the rank deficiency corresponds to the translation ambiguity. In other words, Equation (4) admits a unique solution (up to translation) for fixed lengths, i.e. $\mathcal{F}_{\mathbf{p}}$ is uniquely determined (up to translation) by its graph and measurement function. Combining this observation with the assumption, we get that $\mathcal{F}_{\mathbf{p}}$ is uniquely determined (up to translation and scale) by its graph and directions, i.e. it is globally parallel rigid (and hence parallel rigid).

In simple words, Proposition 4 states that we cannot change the inter-nodal distances of a parallel-rigid point formation independently since, by fixing the length of an edge, we also constrain the length of the remaining edges.

We aim at deriving an algebraic characterization of parallel rigidity in terms of edge lengths, thus we require a linear system having the sole lengths as unknowns. Such system is reported in the following proposition and it involves suitable circuits in $\mathcal{G}$ (see Appendix A for the definition of circuit).

Proposition 5 ( [22]). Let $\mathcal{G}=(\mathcal{V}, \mathcal{E})$ be a connected graph and let $\mathcal{T}$ be a spanning tree of $\mathcal{G}$. For any $e \in \mathcal{E} \backslash \mathcal{T}$ let $\mathbf{c}^{e} \in$ $\{-1,0,1\}^{m}$ denote the circuit obtained by adding $e$ to $\mathcal{T}$, and let $\mathbf{c}_{+}^{e}\left(\mathbf{c}_{-}^{e}\right)$ denote the forward (backward) edges in $\mathbf{c}_{e}$. Let $\left\{\mathbf{u}_{i j}\right\}$ be a set of directions defined on $\mathcal{G}$. A length assignment $\left\{\alpha_{i j}\right\}$ is compatible with edge directions $\left\{\mathbf{u}_{i j}\right\}$, i.e. there exists a point formation $\mathcal{F}_{\mathbf{p}}$ on $\mathcal{G}$ with directions $\mathbf{u}_{i j}$ and lengths $\alpha_{i j}$, if and only if

$$
\sum_{(i, j) \in \mathbf{c}_{+}^{e}} \alpha_{i j} \mathbf{u}_{i j}-\sum_{(i, j) \in \mathbf{c}_{-}^{e}} \alpha_{i j} \mathbf{u}_{i j}=\mathbf{0} \quad \forall e \in \mathcal{E} \backslash \mathcal{T} .
$$

Proof. Note that Equation (44) can be written as

$$
\sum_{(i, j) \in \mathcal{E}}\left[\mathbf{c}^{e}\right]_{i j} \alpha_{i j} \mathbf{u}_{i j}=\mathbf{0} \quad \forall e \in \mathcal{E} \backslash \mathcal{T}
$$

where the circuit $\mathbf{c}^{e}$ is traversed in a cyclic order (clockwise or anti-clockwise), and the (non-zero) entries of $\mathbf{c}^{e}$ have a sign that indicates whether the corresponding edge is traversed along the direction specified by $\mathbf{u}_{i j}$ or not. Equation (45) clearly holds if $\alpha_{i j}=\left\|\mathbf{p}_{j}-\mathbf{p}_{i}\right\|$ and $\mathbf{u}_{i j}=$ $\left(\mathbf{p}_{j}-\mathbf{p}_{i}\right) /\left\|\mathbf{p}_{j}-\mathbf{p}_{i}\right\|$ for a point formation $\mathcal{F}_{\mathbf{p}}$. To prove the opposite direction, we can compute the position of the nodes using the spanning tree $\mathcal{T}$, i.e. the root is set equal to the zero vector and the coordinates of the other nodes are computed via the relation $\mathbf{p}_{j}=\mathbf{p}_{i}+\alpha_{i j} \mathbf{u}_{i j} \Leftrightarrow \mathbf{p}_{j}-\mathbf{p}_{i}=\alpha_{i j} \mathbf{u}_{i j}$. Such point formation has directions equal to $\mathbf{u}_{i j}$ and lengths equal to $\alpha_{i j}$ for all the edges $e \in \mathcal{T}$ (by construction), and also for all the edges $e \in \mathcal{E} \backslash \mathcal{T}$ (due to Equation (45)).

Note that Proposition 5 is about the existence and not the uniqueness of a point formation. For instance, if $\mathcal{G}$ is a tree (which does not contain circuits) and $\left\{\mathbf{u}_{i j}\right\}$ is a given set of directions, then any length assignment is valid, whereas any edge beyond the tree introduces additional constraints. Note also that only one direction in the proof of Proposition 5 requires a connected graph, whereas the other holds for any graph (and also for any set of circuits besides the ones associated with a spanning tree, as observed in Remark 5).

The equations in (45) state that the (signed) sum of directions (weighted with the correct lengths) along circuits must be zero, and they are called the compatibility constraints in [14]. Such equations can be expressed in a compact matrix form if all the lengths $\alpha_{i j}$ are collected in a vector $\boldsymbol{\alpha} \in \mathbb{R}^{m}$ and all the directions $\mathbf{u}_{i j}$ are collected in a matrix $U \in \mathbb{R}^{d \times m}$. Specifically, the equations for a single circuit $\mathbf{c}_{e}$ become

$$
U \operatorname{diag}\left(\mathbf{c}_{e}^{\top}\right) \boldsymbol{\alpha}=\mathbf{0}
$$

or, equivalently, using the Khatri-Rao product

$$
\left(\mathbf{c}_{e}^{\top} \odot U\right) \boldsymbol{\alpha}=\mathbf{0} .
$$

If the equations coming from all the circuits induced by $\mathcal{T}$ are stacked, then a system of $d(m-n+1)$ homogeneous equations is obtained, namely

$$
\left(C_{\mathcal{T}} \odot U\right) \boldsymbol{\alpha}=\mathbf{0}
$$

where $C_{\mathcal{T}} \in\{-1,0,1\}^{(m-n+1) \times m}$ denotes the cycle matrix associated with the circuits $\mathbf{c}_{e}$ for $e \in \mathcal{E} \backslash \mathcal{T}$, which indeed form a fundamental cycle basis (see Appendix A).

The following result states that we can use any cycle basis (fundamental or not) in Equation (48).

Proposition 6. Equation (48) is equivalent to

$$
(C \odot U) \boldsymbol{\alpha}=\mathbf{0}
$$

where $C$ denotes the cycle matrix associated with any cycle basis of $\mathcal{G}=(\mathcal{V}, \mathcal{E})$.

Proof. Let $C_{\mathcal{T}}$ be the cycle matrix associated with the circuits defined in Proposition 5 and let $C$ be the cycle matrix associated with another cycle basis of $\mathcal{G}$. Since the cycle space of the directed graph $\mathcal{G}=(\mathcal{V}, \mathcal{E})$ is a vector space over 
$\mathbb{Q}$, there exists an invertible matrix $R \in \mathbb{Q}^{(m-n+1) \times(m-n+1)}$ such that $C=R C_{\mathcal{T}}$. Using Equation (94) we obtain

$$
C \odot U=\left(R C_{\mathcal{T}}\right) \odot\left(I_{d} U\right)=\left(R \otimes I_{d}\right)\left(C_{\mathcal{T}} \odot U\right) .
$$

Note that the matrix $R \otimes I_{d}$ is invertible (since both $R$ and $I_{d}$ are invertible), hence we get the thesis.

Remark 4. As explained in Appendix A, there are several types of cycle bases for a directed graph $\mathcal{G}=(\mathcal{V}, \mathcal{E})$ besides the fundamental cycle basis, namely zero-one, integral, and undirected cycle bases. In the proof of Proposition 6 a directed cycle basis is used since it generalizes all of them.

Equation (50) means that if a circuit is a linear combination of other circuits, then the compatibility constraint associated with such circuit is a linear combination of the equations associated with the addends. This implies that considering all the circuits in a graph is redundant and what is actually required is a maximal set of independent circuits (i.e. a cycle basis). In summary, we have the following result.

Corollary 2. Let $\mathcal{G}=(\mathcal{V}, \mathcal{E})$ be a connected graph and let $C$ denote the cycle matrix associated with any cycle basis of $\mathcal{G}$. There exists a point formation $\mathcal{F}_{\mathbf{p}}$ on $\mathcal{G}$ with directions $\mathbf{u}_{i j}$ and lengths $\alpha_{i j}$ if and only if

$$
(C \odot U) \boldsymbol{\alpha}=\mathbf{0} .
$$

Remark 5. We observe that Equation (51) can also be derived in an alternative manner. Let us multiply left and right sides in (4) by $\left(C \otimes I_{d}\right)$

$$
\left(C \otimes I_{d}\right)\left(B^{\top} \otimes I_{d}\right) \mathbf{p}=\left(C \otimes I_{d}\right)\left(I_{m} \odot U\right) \boldsymbol{\alpha} .
$$

Using properties (89) and (94) we get

$$
\left(\left(C B^{\top}\right) \otimes I_{d}\right) \mathbf{p}=(C \odot U) \boldsymbol{\alpha} .
$$

Since $C B^{\top}=0$ for any cycle matrix $C$, as stated by Equation (85), the left side vanishes, yielding Equation (51). Note that this computation (which does not require a connected graph) is not a proof of Corollary 2 but it proves only one direction, namely it proves that there exists a point formation with directions $\mathbf{u}_{i j}$ and lengths $\alpha_{i j}$ only if $(C \odot U) \boldsymbol{\alpha}=\mathbf{0}$.

Formula (51) captures at the same time both the structure of the graph (via $C$ ) and the specific values of the directions (via $U$ ). For example, in the case of Figure $4 \mathrm{a}$, the matrix $C \odot U$ has the following structure

$$
C \odot U=\left[\begin{array}{ccccc}
\mathbf{u}_{12} & \mathbf{u}_{24} & \mathbf{u}_{41} & \mathbf{0} & \mathbf{0} \\
\mathbf{0} & -\mathbf{u}_{24} & \mathbf{0} & \mathbf{u}_{23} & \mathbf{u}_{34}
\end{array}\right]
$$

where the following cycle matrix is considered

$$
C=\left[\begin{array}{ccccc}
1 & 1 & 1 & 0 & 0 \\
0 & -1 & 0 & 1 & 1
\end{array}\right]
$$

and the edges are ordered as in

$$
U=\left[\begin{array}{lllll}
\mathbf{u}_{12} & \mathbf{u}_{24} & \mathbf{u}_{41} & \mathbf{u}_{23} & \mathbf{u}_{34}
\end{array}\right] .
$$

A similar formulation is derived in [20]:

$$
D\left(C \otimes I_{d}\right) \operatorname{blockdiag}\left(\left\{\mathbf{u}_{i j}\right\}_{(i, j) \in \mathcal{E}}\right) \boldsymbol{\alpha}=\mathbf{0}
$$

where $D=\left(\left(C \otimes I_{d}\right)\left(C \otimes I_{d}\right)^{\top}\right)^{-1 / 2}$ and blockdiag $\left(\left\{\mathbf{u}_{i j}\right\}_{(i, j) \in \mathcal{E}}\right)$ produces a $d m \times m$ matrix with blocks $\mathbf{u}_{i j}$ along the diagonal. Since
$\left(C \otimes I_{d}\right)$ blockdiag $\left(\left\{\mathbf{u}_{i j}\right\}_{(i, j) \in \mathcal{E}}\right)=C \odot U$ and $D$ is invertible we get

$$
\operatorname{ker}\left(D\left(C \otimes I_{d}\right) \operatorname{blockdiag}\left(\left\{\mathbf{u}_{i j}\right\}_{(i, j) \in \mathcal{E}}\right)\right)=\operatorname{ker}(C \odot U)
$$

which means that Equation (57) is equivalent to Equation (51). The latter enjoys a more compact formulation, which permits us to exploit algebraic properties of the Khatri-Rao product, as done (e.g.) in the proof of Proposition 6.

Hereafter we use the notation $U$ to denote the $d \times m$ matrix constructed from any set of directions, and we use the notation $U_{\mathbf{p}}$ to denote the $d \times m$ matrix built from the directions of a point formation $\mathcal{F}_{\mathbf{p}}$. Note that if $\mathcal{F}_{\mathbf{p}}$ is a point formation then Equation (51) holds, i.e. the null-space of $C \odot U_{\mathbf{p}}$ is at least 1-dimensional. The following theorem, which is a direct consequence of Proposition 4 and Corollary 2 , states such null-space is exactly 1-dimensional if and only if $\mathcal{F}_{\mathbf{p}}$ is parallel rigid.

Theorem 7. Let $\mathcal{G}=(\mathcal{V}, \mathcal{E})$ be a connected graph and let $C$ denote the cycle matrix associated with any cycle basis of $\mathcal{G}$. A point formation $\mathcal{F}_{\mathbf{p}}$ on $\mathcal{G}$ is parallel rigid if and only if

$$
\operatorname{dim}\left(\operatorname{ker}\left(C \odot U_{\mathbf{p}}\right)\right)=1
$$

or, equivalently, if and only if

$$
\operatorname{rank}\left(C \odot U_{\mathbf{p}}\right)=m-1 .
$$

Remark 6. Although the focus of this section is the localizability aspect, it also provides a way to solve bearingbased localization via an edge-based approach. More precisely, given a set of directions collected in a matrix $U$, the localization problem can be solved in two-steps:

1) the unknown scales contained in $\boldsymbol{\alpha} \in \mathbb{R}^{m}$ are recovered as the solution to the homogeneous linear system (51);

2) the unknown node locations contained in $\mathbf{p} \in \mathbb{R}^{d n}$ are derived as the solution to the non-homogeneous linear system (4) with known $\boldsymbol{\alpha}$.

It is assumed here that bearing-based localization admits a unique solution (up to translation and scale). The existence of a solution is equivalent to assume that the input directions are noise free, or, equivalently, that there exists a point formation $\mathcal{F}_{\mathbf{p}}$ whose directions are those in $U$, i.e. $U=U_{\mathbf{p}}$. The uniqueness of the solution is equivalent to assume that such point formation is parallel rigid, as explained in Section 2.1.3, which coincides with assuming that the nullspace of $(C \odot U)$ is 1-dimensional, according to Theorem 7. This implies that Step 1 returns a unique solution (up to scale). As concerns Step 2, the solution is unique (up to translation) if the graph is connected, as explained in the proof of Proposition 4. As a matter of fact, Step 2 is an instance of group synchronization [40] in $\mathbb{R}^{3}$.

Experiments reported in [40] suggest that the approach outlined Remark 6 is comparable in accuracy to state-of-theart algorithms which exploit the node-based formulation, thus showing that both node-based and edge-based algorithms are viable in practice. 


\subsection{Generic Rigidity}

We now consider the property of generic rigidity. Due to Theorem 7 we can say that a connected graph $\mathcal{G}=(\mathcal{V}, \mathcal{E})$ is generically parallel rigid in $d$-space if and only if $\operatorname{rank}(C \odot$ $\left.U_{\mathbf{p}}\right)=m-1$ or, equivalently, if and only if $\operatorname{dim}(\operatorname{ker}(C \odot$ $\left.\left.U_{\mathbf{p}}\right)\right)=1$, where $U_{\mathbf{p}}$ is constructed using a generic point formation $\mathcal{F}_{\mathbf{p}}$ defined on $\mathcal{G}$. Reasoning in the same way as in Section 2.2, we get the following algebraic characterization of generic parallel rigidity in terms of cycles in the graph.

Theorem 8. A connected graph $\mathcal{G}=(\mathcal{V}, \mathcal{E})$ is generically parallel rigid in $d$-space if and only if

$$
\max _{p \in \mathbb{R}^{n d}} \operatorname{rank}\left(C \odot U_{\mathbf{p}}\right)=m-1,
$$

or, equivalently, if and only if

$$
\min _{p \in \mathbb{R}^{n d}} \operatorname{dim}\left(\operatorname{ker}\left(C \odot U_{\mathbf{p}}\right)\right)=1 .
$$

Similarly to the node-based case [19], Theorem 8 can be used to develop a randomized test for checking generic parallel rigidity, where a matrix $U_{\mathbf{p}}$ is built from a point formation randomly sampled from i.i.d. Gaussian distribution. Theorem 8 gives also rise to an algorithm to identify maximal rigid components of flexible graphs [20], where the null-space of $C \odot U_{\mathbf{p}}$ is computed and sets of parallel lines among the rows are identified. Theoretical results on the topology of maximal rigid components can be found in [41]. The authors of [41] also study the rigidity recovery problem, where the goal is to turn a flexible graph into a rigid one by adding new edges, and they provide both a combinatorial algorithm and a greedy strategy to select which edges to add.

Note that in order to guarantee that Equation (61) holds, the number of rows in $C \odot U_{\mathbf{p}}$ must be greater than (or equal to) $m-1$, i.e. the following necessary condition must be satisfied

$$
d(m-n+1) \geqslant m-1
$$

which is equivalent to Equation (34).

The formulation of Theorem 8 , although equivalent to the node-based one, enables us to prove results involving the topology of the graph, showing, for instance, why triangulated graphs are rigid while graphs with long cycles may loose this property. Let us start by presenting a necessary condition for generic parallel rigidity, namely biconnectivity (see Appendix A for the definition of biconnected graph and related properties). This was also mentioned en-passant in [34].

Proposition 7. If a graph $\mathcal{G}=(\mathcal{V}, \mathcal{E})$ is generically parallel rigid in $d$-space, then it is biconnected.

Proof. If $\mathcal{G}=(\mathcal{V}, \mathcal{E})$ is not biconnected then it can be partitioned into biconnected components. Let $b>1$ denotes the number of such components and let $\mathcal{E}^{1}, \ldots, \mathcal{E}^{b}$ denote their edge sets. Since the set of biconnected components induces a partition of the edge set $\mathcal{E}$ and a circuit belongs to only one biconnected component, the matrix $C \odot U_{\mathbf{p}}$ can be expressed as a block-diagonal matrix (up to a re-ordering of the edges), where each block corresponds to a biconnected component, namely $C \odot U_{\mathbf{p}}=\operatorname{blockdiag}\left(G_{1}, \ldots, G_{b}\right)$. Let us assume that all such components are generically parallel rigid, otherwise the thesis is obvious, thus in each component the lengths of a generic point formation are uniquely determined by its graph and directions (up to a global scale), namely $\operatorname{rank}\left(G_{i}\right)=\left|\mathcal{E}^{i}\right|-1$. Thus $\operatorname{rank}\left(C \odot U_{\mathbf{p}}\right)=$ $\sum_{i=1}^{b}\left(\left|\mathcal{E}^{i}\right|-1\right)=m-b<m-1$, meaning that all such scales can not be reconciled into a single scale, hence $\mathcal{G}$ is generically flexible.

It is straightforward to see that the necessary condition of Proposition 7 alone is not sufficient: for instance, the graph associated with the point formation in Figure $2 \mathrm{a}$ is biconnected and flexible in 2-space. However, Proposition 7 gives a simple condition to detect non-rigid graphs: for instance, it can be established that the graphs reported in Figure 5 are flexible in $d$-space.

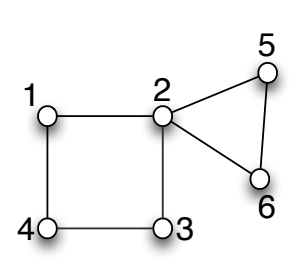

(a)

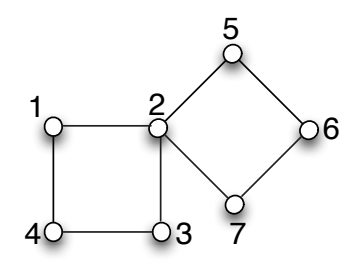

(b)
Figure 5: Non biconnected graphs.

Note that if $\mathcal{G}=(\mathcal{V}, \mathcal{E})$ is biconnected (and contains at least 3 vertices), then it is bridgeless (see Appendix A), i.e. each edge in $\mathcal{E}$ belongs to (at least) one cycle (and hence one circuit). This implies that if $\mathcal{G}$ is generically parallel rigid in $d$-space, then it is bridgeless. This result is not surprising since an edge not belonging to any circuit is not constrained by the other edges, and hence its length can be chosen arbitrarily. Such an edge corresponds to a column of zeros in $C$, which makes the rank of $C \odot U_{\mathbf{p}}$ drop. Two examples of graphs with a bridge are reported in Figure 6.

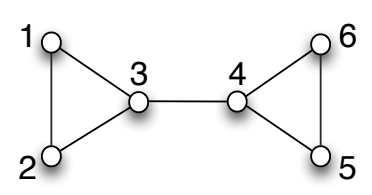

(a)

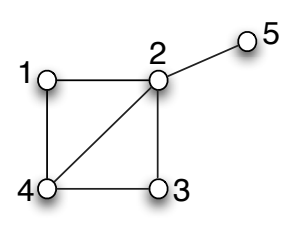

(b)
Figure 6: Non bridgeless graphs.

We now consider the case where $\mathcal{G}$ consists of a single circuit of length $\ell \geqslant 3$, and show that short circuits are rigid while long circuits are flexible. In this case $m=n=\ell$, hence Equation (63) rewrites $\ell \leqslant d+1$, i.e. the following proposition holds.

Proposition 8 ( [14], [20]). A circuit of length $\ell \geqslant d+2$ is not generically parallel rigid in d-space.

What happens for circuits of length $\ell \leqslant d+1$ ? It can be shown that such circuits are generically parallel rigid in $\mathbb{R}^{d}$. Figure 7 reports some examples in $\mathbb{R}^{3}$.

Proposition 9. A circuit of length $\ell \leqslant d+1$ is generically parallel rigid in $d$-space. 
Proof. If $\mathcal{G}$ consists of a single circuit then $C \odot U_{\mathbf{p}}$ is a $d \times$ $\ell$ matrix where each column contains one direction (with the correct sign). Since a given point formation $\mathcal{F}_{\mathbf{p}}$ satisfies Equation (51) then

$$
\operatorname{rank}\left(C \odot U_{\mathbf{p}}\right) \leqslant \ell-1
$$

which means that the points in $\mathcal{F}_{\mathbf{p}}$ belong to an affine subspace of $\mathbb{R}^{d}$ of dimension at most $\ell-1$. Note that specific configurations make $\operatorname{rank}\left(C \odot U_{\mathbf{p}}\right)$ drop. Specifically, $\operatorname{rank}\left(C \odot U_{\mathbf{p}}\right)=1$ if and only if the points in $\mathcal{F}_{\mathbf{p}}$ lie on a common line; $\operatorname{rank}\left(C \odot U_{\mathbf{p}}\right)=2$ if and only if the points in $\mathcal{F}_{\mathbf{p}}$ lie on a common plane; $\ldots \operatorname{rank}\left(C \odot U_{\mathbf{p}}\right)=\ell-2$ if and only if the points in $\mathcal{F}_{\mathbf{p}}$ lie on an affine subspace of $\mathbb{R}^{d}$ of dimension $\ell-2$. On the contrary, if the points in $\mathcal{F}_{\mathbf{p}}$ are generic, then $\operatorname{rank}\left(C \odot U_{\mathbf{p}}\right)=\ell-1$, meaning that $\mathcal{F}_{\mathbf{p}}$ (and hence $\mathcal{G}$ ) is parallel rigid in $\mathbb{R}^{d}$.

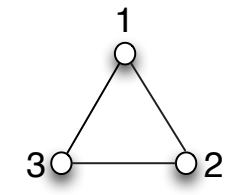

(a) Parallel rigid

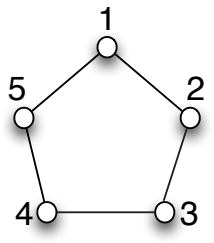

(c) Flexible

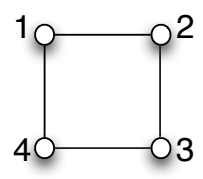

(b) Parallel rigid

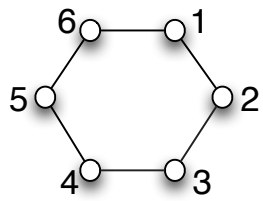

(d) Flexible
Figure 7: Rigidity of circuits in 3-space.

Remark 7. Note that there is a key difference between a circuit of length $\ell=d+1$ and a circuit of length $\ell \leqslant d$, which is essential to understand the next section. In the $\ell=d+1$ case, $C \odot U_{\mathbf{p}}$ is a $d \times(d+1)$ matrix, thus its rank is at most $d=\ell-1$ independently of the directions, i.e. Equation (64) is satisfied even if $U_{\mathbf{p}}$ is substituted by a random set of $d+1$ directions. On the contrary, in the $\ell \leqslant d$ case, if we take a random set of $\ell$ directions then Equation (64) will not be satisfied.

Propositions 8 and 9 completely characterize the localizability of a graph made of a single circuit (in terms of its length). What happens to graphs made of several circuits? The remainder of this section reports sufficient conditions for parallel rigidity, which give some insights on how to answer such question.

Given a cycle basis for a (connected) graph $\mathcal{G}=(\mathcal{V}, \mathcal{E})$, the cycle graph ${ }^{2} \mathcal{G}_{C}$ is defined as follow: each vertex corresponds to a circuit in the basis, and an edge is present between two vertices if and only if the corresponding circuits share (at least) one edge in $\mathcal{G}$. The notion of cycle graph is exploited by the following theorem.

2. This notion generalizes the "triplet graph" of [42].
Theorem 9. Let $\mathcal{G}=(\mathcal{V}, \mathcal{E})$ be a connected bridgeless graph. Suppose that there exists a cycle basis of $\mathcal{G}$ such that the following conditions are satisfied:

1) each circuit in the basis has length at most $d+1$;

2) the associated cycle graph $\mathcal{G}_{C}$ is connected.

Then $\mathcal{G}$ is generically parallel rigid in $d$-space.

Proof. Each circuit in the basis is generically parallel rigid in $d$-space due to Proposition 9. Since the cycle graph is connected by assumption, we can start with any circuit and reach all the others through a path, thus producing a growing rigid subgraph. Specifically, let us consider a node in $\mathcal{G}_{C}$ (i.e. a circuit) and let us take an edge incident to such node (i.e. a circuit sharing one edge with the first circuit). The union of these circuits is rigid since both of them are rigid and they have one edge in common. We can repeat this line of reasoning considering all the remaining circuits, obtaining a parallel rigid subgraph. Such subgraph coincides with $\mathcal{G}$ itself since $\mathcal{G}$ is bridgeless, and hence each edge in $\mathcal{E}$ belongs to (at least) one circuit in the basis.

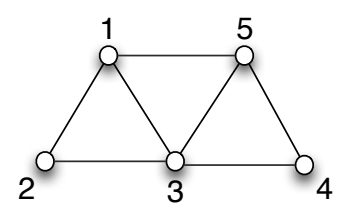

(a)

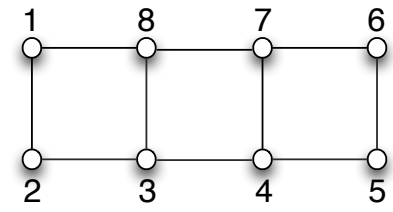

(b)

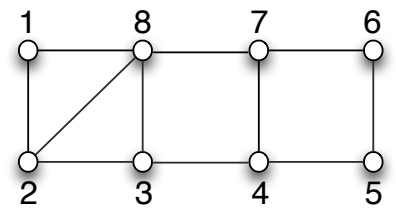

(c)

Figure 8: Examples of graphs which satisfy the assumptions in Theorem 9, and hence they are generically parallel rigid in d-space, with $d \geqslant 2$ (a) and $d \geqslant 3(\mathrm{~b}, \mathrm{c})$.

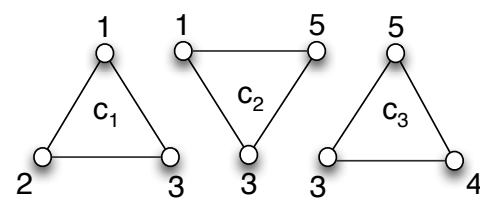

(a)

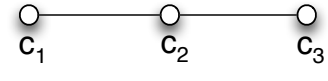

(b)
Figure 9: Left: cycle basis composed of 3-length circuits for the graph in Figure 8a. Right: cycle graph $\mathcal{G}_{C}$ associated with such cycle basis.

By means of Theorem 9 it can be established that the graphs in Figure 8 are parallel rigid in d-space, with $d \geqslant 2$ (Figure $8 \mathrm{a}$ ) and $d \geqslant 3$ (Figures $8 \mathrm{~b}$ and $8 \mathrm{c}$ ). The graph in $8 \mathrm{a}$ admits a cycle basis composed of three 3-length circuits, the graph in Figure 8b admits a cycle basis composed of 
three 4-length circuits, and the graph in Figure 8c admits a cycle basis composed of two 3-length circuits, and two 4length circuits. In all these cases the associated cycle graph is connected. The one associated with Figure 8a is reported in Figure 9.

Remark 8. Note that the reverse of Theorem 9 is not true. For instance, the graph reported in Figure 10 is parallel rigid in 3-space but Condition 1 can not be fulfilled. Let us consider the following cycle basis

$$
\begin{aligned}
\mathbf{c}_{1} & =\left[\begin{array}{lllllllll}
1 & 1 & 1 & 0 & 0 & 0 & 0 & 0 & 0
\end{array}\right]^{\top} \\
\mathbf{c}_{2} & =\left[\begin{array}{lllllllll}
0 & 1 & 0 & 1 & 1 & 1 & 0 & 0 & 0
\end{array}\right]^{\top} \\
\mathbf{c}_{3} & =\left[\begin{array}{lllllllll}
1 & 0 & 0 & 0 & 0 & -1 & 1 & 1 & 1
\end{array}\right]^{\top}
\end{aligned}
$$

where the edges are ordered as in

$U_{\mathbf{p}}=\left[\begin{array}{lllllllll}\mathbf{u}_{12} & \mathbf{u}_{26} & \mathbf{u}_{61} & \mathbf{u}_{67} & \mathbf{u}_{73} & \mathbf{u}_{32} & \mathbf{u}_{34} & \mathbf{u}_{45} & \mathbf{u}_{51}\end{array}\right]$

Note that the associated cycle graph $\mathcal{G}_{C}$ is connected. It is easy to see that cycle bases composed of shorter circuits do not exist, thus Condition 1 can not be satisfied. To prove that $\mathcal{G}$ is generically parallel rigid we consider a generic point formation $\mathcal{F}_{\mathbf{p}}$ and show that its lengths are uniquely determined by its graph and directions, i.e. Equation (51) admits a unique solution (up to scale). Note that Equation (51) rewrites

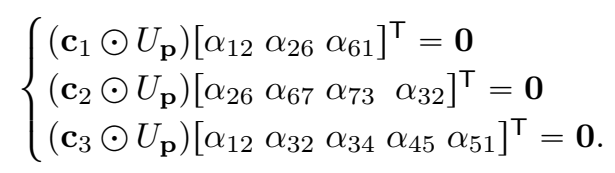

Instead of solving Equation (67) globally, we follow a sequential approach. Let us start with the 3-length circuit $\mathbf{c}_{1}$ : its lengths are uniquely determined (up to a global scale) since it is parallel rigid, meaning that, if we arbitrarily fix the value of (e.g.) $\alpha_{12}$, we can (uniquely) compute the remaining lengths (i.e. $\alpha_{26}$ and $\alpha_{61}$ ) by solving the first row in Equation (67). Then we use the obtained value of $\alpha_{26}$ to fix the global scale of the 4-length circuit $\mathbf{c}_{2}$ (which is parallel rigid), and (uniquely) solve for the remaining scales (i.e. $\alpha_{67}, \alpha_{73}$ and $\alpha_{32}$ ) by considering the second row in Equation (67). Note that this is possible since $\mathbf{c}_{1}$ and $\mathbf{c}_{2}$ share an edge. Finally, we consider the 5-length circuit $\mathbf{c}_{3}$, which is flexible (if considered in isolation). However, the key observation is that the values of $\alpha_{12}$ and $\alpha_{32}$ have been already computed, thus only three unknowns remain. In other words, the fourth row in Equation (67) becomes equivalent to the compatibility constraint of a 4-length circuit, and hence the remaining lengths (i.e. $\alpha_{34}, \alpha_{45}$ and $\alpha_{51}$ ) are uniquely determined. In this way we are able to compute all the unknowns up to a single scale, which corresponds to the arbitrary choice of $\alpha_{12}$, meaning that $\mathcal{G}$ is parallel rigid.

Note that the sequential approach outlined in Remark 8 heavily depends on the chosen cycle basis and on the order in which circuits are processed. On the contrary, if Equation (67) is solved globally, then any cycle basis can be used, due to Proposition 6.

Remark 8 has pointed out that a long circuit (which is flexible alone) can be part of a larger rigid graph. Indeed, if the compatibility constraint of a flexible circuit is properly

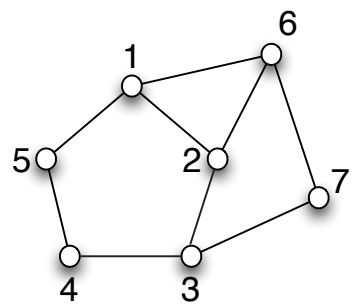

Figure 10: Example of a rigid graph in 3-space which does not satisfy the assumptions in Theorem 9. It admits a cycle basis composed of one 3-length circuit, one 4-length circuit and one 5-length circuit, and cycle bases with shorter circuits do not exist.

combined with those of rigid circuits, it may results in a system with a unique solution (up to scale).

We conclude this section by stating the following result, which is a generalization of Theorem 9.

Theorem 10. Let $\mathcal{G}=(\mathcal{V}, \mathcal{E})$ be a connected bridgeless graph. Suppose that the circuits of a cycle basis can be ordered such that the first circuit has length at most $d+1$, and each of the remaining circuits satisfies one of the following conditions:

1) it has length at most $d+1$ and it has at least one edge in common with the subgraph induced by the previous ones;

2) it has length at least $d+2$, and it has at least one edge in common and at most $d$ edges not in common with the subgraph induced by the previous ones.

Then $\mathcal{G}$ is generically parallel rigid in d-space.

Proof. Let $\mathbf{c}_{1}, \ldots, \mathbf{c}_{r}$ denote the circuits in the basis (ordered as in the assumptions) with $r=m-n+1$. Note that the cycle graph $\mathcal{G}_{C}$ is connected. The first circuit $\mathbf{c}_{1}$ is parallel rigid in $\mathrm{d}$-space since it has length at most $d+1$. Let us consider the second circuit $\mathbf{c}_{2}$. If Condition 1 is satisfied, then the union of $\mathbf{c}_{1}$ and $\mathbf{c}_{2}$ is rigid, since both circuits are rigid and they have (at least) one edge in common. If Condition 2 is satisfied, then we can use the same argument as in Remark 8 and prove that $\mathbf{c}_{2}$ is equivalent to a circuit of length (at most) $d+1$ with one edge in common with $\mathbf{c}_{1}$, hence their union is rigid. Indeed, given a generic point formation $\mathcal{F}_{\mathbf{p}}$, we can first solve the compatibility constraint associated with $\mathbf{c}_{1}$, and arbitrarily fix the length of an edge in $\mathbf{c}_{1}$ in order to fix the global scale, thus all the lengths of $\mathcal{F}_{\mathbf{p}}$ in $\mathbf{c}_{1}$ are uniquely determined. Then, thanks to the edges in common with $\mathbf{c}_{1}$, (at most) $d$ unknowns remain when considering the compatibility constraint associated with $\mathbf{c}_{2}$, which can be computed as in a circuit of length (at most) $d+1$ where one length is fixed. We can repeat this line of reasoning considering the remaining circuits $\mathbf{c}_{3}, \ldots, \mathbf{c}_{r}$ one after the other, obtaining a growing parallel rigid subgraph. Such subgraph coincides with $\mathcal{G}$ itself since $\mathcal{G}$ is bridgeless.

It is easy to see that the graph reported in Figure 10 satisfies the conditions in Theorem 10. Establishing whether such conditions are also necessary is subject of future research. 


\section{The Parallel Rigidity Index}

The results on generic rigidity reported in Sections 2 and 3 are derived considering a generic point formation $\mathcal{F}_{\mathrm{p}}$, which uniquely defines a set of directions (i.e. a measurement function). What happens if the directions, not the coordinates of the points in $\mathcal{F}_{\mathbf{p}}$, are generic? This issue is addressed in [18], where the authors, starting from the edge-based formulation, introduce the concept of parallel rigidity index, which is a property of the graph and dimension $d$.

Definition 7. The parallel rigidity index $\mathcal{I}_{d}(\mathcal{G})$ of a connected graph $\mathcal{G}=(\mathcal{V}, \mathcal{E})$ in $d$-space is defined as

$$
\mathcal{I}_{d}(\mathcal{G})=\min _{U \in \mathbb{R}^{d \times m}} \operatorname{dim}(\operatorname{ker}(C \odot U)) .
$$

Note that there is an essential difference between the right side in Equation (68) and the left side in Equation (62): the former computes the minimum over all the possible edge directions (contained in $U$ ) whereas the latter computes the minimum over all possible point formations $\mathcal{F}_{\mathbf{p}}$ (which define a matrix $U_{\mathbf{p}}$ ).

Remark 9. An equivalent definition for the parallel rigidity index is the following

$$
\mathcal{I}_{d}(\mathcal{G})=m-\max _{U \in \mathbb{R}^{d \times m}} \operatorname{rank}(C \odot U) .
$$

We observe that the above equation does not coincide, in general, with $m-\operatorname{ger}(C \odot U)$, where $\operatorname{ger}(C \odot U)$ denotes the generic rank [43] of $C \odot U$, that is the maximal rank that $C \odot U$ (viewed as a structured matrix) achieves as a function of its arbitrary (non-zero) elements. Indeed, when considering Equation (69), the nonzero entries in $(C \odot U)$ are not arbitrary at all: for instance, if an edge $(i, j)$ belongs to more than one circuit in $C$, then multiple copies of $\mathbf{u}_{i j}$ appear in $C \odot U$, one for each circuit. It is easy to see that

$$
\mathcal{I}_{d}(\mathcal{G}) \geqslant m-\operatorname{ger}(C \odot U) .
$$

The generic rank has also a combinatorial description [43], namely it is equal to the maximum number of edges of any matching of a bipartite graph constructed as follows: nodes correspond to rows/columns of the matrix, and edges correspond to its nonzero entries. Establishing under which conditions (if any) equality holds in (70) is left to future research.

It can be shown that the minimum in Equation (68) is attained for generic directions [18], where a set of directions is called generic if its $d n$ coordinates are not algebraically dependent. Thus we can rewrite the parallel rigidity index as $\mathcal{I}_{d}(\mathcal{G})=\operatorname{dim}(\operatorname{ker}(C \odot U))$, or, equivalently,

$$
\mathcal{I}_{d}(\mathcal{G})=m-\operatorname{rank}(C \odot U)
$$

where $U$ is a $d \times m$ matrix containing generic directions in its columns. This suggests a randomized procedure to compute the parallel rigidity index, where a matrix $U$ is built from a set of directions sampled at random on the sphere in $d$ space, similarly to the randomized test for parallel rigidity proposed in [19].

As observed in Section 3, a point formation $\mathcal{F}_{\mathbf{p}}$ satisfies Equation (51), i.e. the null-space of $C \odot U_{\mathbf{p}}$ is at least 1dimensional. On the contrary, if we consider generic directions we can not expect to find a non-trivial solution to
$(C \odot U) \boldsymbol{\alpha}=\mathbf{0}$ for any graph $\mathcal{G}$, i.e. it may happen that a point formation on $\mathcal{G}$ with such directions does not exist. In other words, the parallel rigidity index $\mathcal{I}_{d}(\mathcal{G})$ can be equal to zero, which means that the only length assignment compatible with a generic set of $m$ directions is $\boldsymbol{\alpha}=\mathbf{0}$, i.e. all the nodes collapse into one point in $\mathbb{R}^{d} \cdot \mathcal{I}_{d}(\mathcal{G})=1$ means that there exists a unique (up to scale) length assignment $\boldsymbol{\alpha} \neq \mathbf{0}$ compatible with a generic set of directions, i.e. for any set of directions in $\mathbb{R}^{d}$ (with coordinates not algebraically dependent) there exists a unique (up to translation and scale) point formation $\mathcal{F}_{\mathbf{p}}$ on $\mathcal{G}$ (such that not all the nodes are coincident) having such directions as measurement function. $\mathcal{I}_{d}(\mathcal{G}) \geqslant 2$ means that there exists a point formation $\mathcal{F}_{\mathbf{p}}$ on $\mathcal{G}$ compatible with a generic set of directions, but it is not unique (up to translation and scale), i.e. there are additional degrees of freedom.

Using the definition, we can easily compute the parallel rigidity index of a graph $\mathcal{G}$ consisting of a single circuit of length $\ell \geqslant 3$. The difference between the $\ell \leqslant d$ case and the $\ell=d+1$ case has been already observed in Remark 7 .

Proposition 10. Let $\mathcal{G}$ be a circuit of length $\ell$.

- If $\ell \leqslant d$ then $\mathcal{I}_{d}(\mathcal{G})=0$;

- if $\ell=d+1$ then $\mathcal{I}_{d}(\mathcal{G})=1$;

- if $\ell \geqslant d+2$ then $\mathcal{I}_{d}(\mathcal{G})=\ell-d \geqslant 2$.

Proof. If $\mathcal{G}$ is a circuit of length $\ell$, then $m=\ell$ and $C \odot U$ is a $d \times \ell$ matrix containing any (generic) direction in each column. Thus $C \odot U$ has full rank and, using (71), we get $\mathcal{I}_{d}(\mathcal{G})=\ell-\min \{d, \ell\}$.

Using Proposition 10, we get that $\mathcal{I}_{3}(\mathcal{G})=0$ for the graph in Figure $7 \mathrm{a}, \mathcal{I}_{3}(\mathcal{G})=1$ for the graph in Figure $7 \mathrm{~b}$, $\mathcal{I}_{3}(\mathcal{G})=2$ for the graph in Figure $7 \mathrm{c}$, and $\mathcal{I}_{3}(\mathcal{G})=3$ for the graph in Figure $7 \mathrm{~d}$.

The following result provides a combinatorial characterization of the parallel rigidity index of a connected graph.

Theorem 11 ( [18]). The parallel rigidity index $\mathcal{I}_{d}(\mathcal{G})$ of a connected graph $\mathcal{G}=(\mathcal{V}, \mathcal{E})$ in $d$-space is equal to the minimal size of the intersection of $d$ spanning trees of $\mathcal{G}$.

Corollary 3. Let $\mathcal{G}=(\mathcal{V}, \mathcal{E})$ be a connected graph, let $n_{b}$ denotes the number of bridges, and let $\mathcal{G}^{1}, \ldots, \mathcal{G}^{n_{b}}$ denote the connected components obtained after removing all the bridges from $\mathcal{G}$. Then

$$
\mathcal{I}_{d}(\mathcal{G})=n_{b}+\sum_{i=1}^{n_{b}} \mathcal{I}_{d}\left(\mathcal{G}^{i}\right)
$$

Proof. Note that, if $\mathcal{G}$ has a bridge, then this bridge belongs to any spanning tree of $\mathcal{G}$. Recall that the removal of a bridge disconnects the graph, and hence it can be partitioned into connected components. Thus any minimal intersection of $d$ spanning trees of $\mathcal{G}$ is the union of all the bridges and a minimal intersection of $d$ spanning trees for each connected component.

According to Theorem 11, the parallel rigidity index can be computed by first counting the edges in common between $d$ (distinct) spanning trees, and then taking the minimum over all the possible choices of such spanning trees. In this way it can be established, for instance, that 
$\mathcal{I}_{d}(\mathcal{G})=0$ for the graphs in Figures $4 \mathrm{a}$ and $8 \mathrm{a}$. Using Corollary 3 we get that $\mathcal{I}_{d}(\mathcal{G})=1$ for the graphs in Figure 6. In general, $\mathcal{I}_{d}(\mathcal{G})$ is greater than or equal to the number of bridges of $\mathcal{G}$. Note that adding an edge between existing nodes may modify the parallel rigidity index. For instance, as it can be easily verified, the graph in Figure $8 \mathrm{~b}$ satisfies $\mathcal{I}_{3}(\mathcal{G})=1$ whereas the graph in Figure $8 \mathrm{c}$ (which is obtained from the former by adding one edge) satisfies $\mathcal{I}_{3}(\mathcal{G})=0$.

The following result, which is a straightforward consequence of Theorem 11, establishes the relation between the parallel rigidity index in $d$-space and in $(d+1)$-space.

Corollary 4 ( [18]). The parallel rigidity index $\mathcal{I}_{d}(\mathcal{G})$ of a connected graph $\mathcal{G}=(\mathcal{V}, \mathcal{E})$ in d-space decreases as $d$ grows, namely $\mathcal{I}_{d}(\mathcal{G}) \geqslant \mathcal{I}_{d+1}(\mathcal{G})$.

What is the relation between the parallel rigidity index and the generic rigidity of a graph? Since the set of directions coming from point formations is contained in the set of all possible directions, we get

$$
\min _{U \in \mathbb{R}^{d \times m}} \operatorname{dim}(\operatorname{ker}(C \odot U)) \leqslant \min _{p \in \mathbb{R}^{n d}} \operatorname{dim}\left(\operatorname{ker}\left(C \odot U_{\mathbf{p}}\right)\right) .
$$

Thus, if $\mathcal{I}_{d}(\mathcal{G}) \geqslant 2$ then $\mathcal{G}$ is generically flexible in $d$-space. In other words, if $\mathcal{G}$ is generically parallel rigid in $d$-space, then either $\mathcal{I}_{d}(\mathcal{G})=0$ or $\mathcal{I}_{d}(\mathcal{G})=1$. The converse is not true, i.e. the parallel rigidity index of a flexible graph can assume any value. For example, the graphs reported in Figure 5 are both flexible in 3-space and - as it can be easily verified the parallel rigidity index is $\mathcal{I}_{d}(\mathcal{G})=1$ for the left sub-figure and $\mathcal{I}_{d}(\mathcal{G})=2$ for the right sub-figure. The flexible graph in Figure $4 \mathrm{~b}$ satisfies $\mathcal{I}_{d}(\mathcal{G})=0$. More examples are reported in Figure 11.

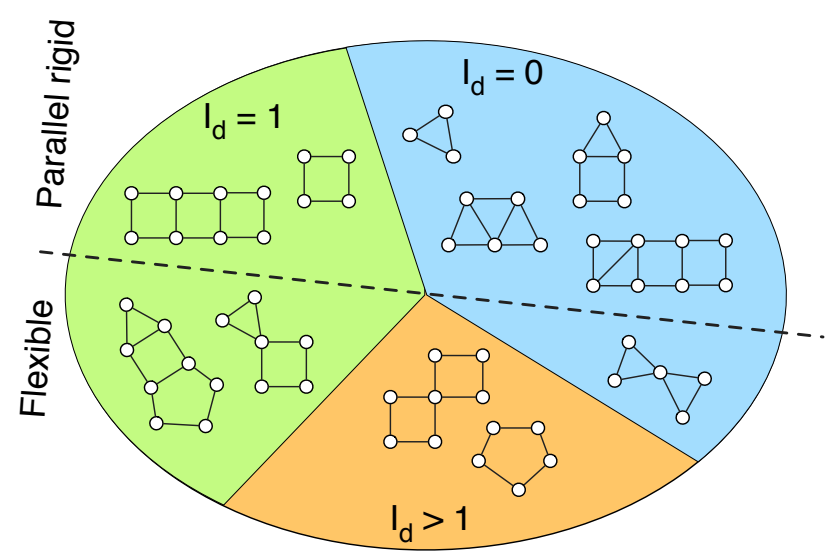

Figure 11: Relationship between the notion of generic parallel rigidity and the parallel rigidity index $\mathcal{I}_{d}(\mathcal{G})$ with $d=3$. The graphs amenable to error compensation are the ones in the upper right corner.

\subsection{Which rigidity for error compensation?}

Let us now come back to the network localization problem. As explained in Remark 6, given a set of directions $\left\{\mathbf{u}_{i j}\right\}$ and a graph $\mathcal{G}=(\mathcal{V}, \mathcal{E})$, the unknown node locations $\mathbf{p}_{i} \in \mathbb{R}^{d}$ can be recovered via a two-step procedure: first, the unknown lengths $\alpha_{i j}$ are computed by solving system (51); then, the unknown node locations are derived as the solution of Equation (4). Other methods can also be found in the literature, which refer to the node-based formulation (e.g. [30], [32]).

Let us consider the noiseless case where $\mathbf{u}_{i j}=\left(\mathbf{p}_{j}-\right.$ $\left.\mathbf{p}_{i}\right) /\left\|\mathbf{p}_{j}-\mathbf{p}_{i}\right\|$, with $\mathbf{p}$ generic. In this scenario the graph is required to be generically parallel rigid in $d$-space, in order to guarantee that Equation (51) has a unique solution (up to scale), and hence the network localization problem, i.e. Equation (4), admits a unique solution (up to translation and scale).

Suppose now that measurements are noisy (as it is always the case in practice), i.e. $\mathbf{u}_{i j} \approx\left(\mathbf{p}_{j}-\mathbf{p}_{i}\right) /\left\|\mathbf{p}_{j}-\mathbf{p}_{i}\right\|$. In this case, besides parallel rigidity, one has a further requirement, which is related to error compensation. In particular, one wants to exclude graphs that, by virtue of their structure, always yield an exact solution to the network localization problem regardless of the direction measures. This analysis cannot be performed within the generic rigidity framework, for it is based on generic point formations (which always yield exact directions), but the parallel rigidity index is required, which relies on generic directions.

As a matter of fact, the graphs which, due to their structure, satisfy $\operatorname{dim}(\operatorname{ker}(C \odot U))=1$ for any (generic) $U$ are all those and only those with $\mathcal{I}_{d}(\mathcal{G})=1$, thus there exists an exact solution to Equation (51) in the presence of noise and/or outliers. On the contrary, $\mathcal{I}_{d}(\mathcal{G})=0$ means that $\operatorname{dim}(\operatorname{ker}(C \odot U))=0$ and no exact solution to Equation (51) exists (apart from the trivial solution $\boldsymbol{\alpha}=\mathbf{0}$ ), as one expects in a noisy case.

We conclude that: among all the graphs which are generically parallel rigid in $d$-space, only the ones with $\mathcal{I}_{d}(\mathcal{G})=0$ are amenable to error compensation. They correspond to the upper right corner of Figure 11.

A similar analysis is performed in [44] for distance-based localization, where the authors define two rigidity indexes that permit them to identify graphs with better capacity than others to recover node locations in the presence of noise.

\section{Relation to Structure from Motion}

As observed also in [19], a relevant topic in Computer Vision which is closely related to bearing-based network localization is structure from motion [5], that is the problem of recovering both the structure of the scene (i.e. 3D coordinates of scene points) and camera motion (i.e. angular attitudes and positions of the cameras), given a collection of images.

A paradigm which is receiving a growing attention in the community is represented by synchronization [40] or averaging [46] methods (e.g. [32], [33], [45], [47], [48], [49]), where the motion part is decoupled from structure and solved first. These method are usually faster and less memorydemanding than sequential and hierarchical methods (e.g. [50], [51], [52], [53], [54]), and they ensure a fair distribution of the errors among the cameras, being global.

As for the motion task, most of these techniques first estimate the angular attitudes of the cameras and then recover their positions, starting from relative motions derived from point correspondences. We are concerned here with the second step only, namely computing camera positions. 


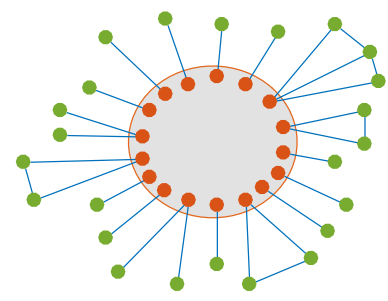

(a) Madrid Metropolis

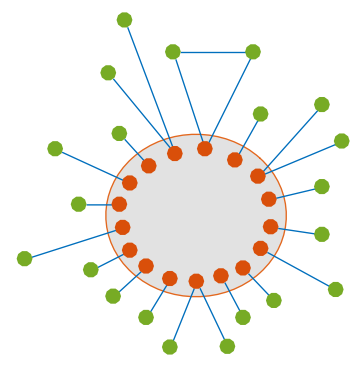

(b) Alamo

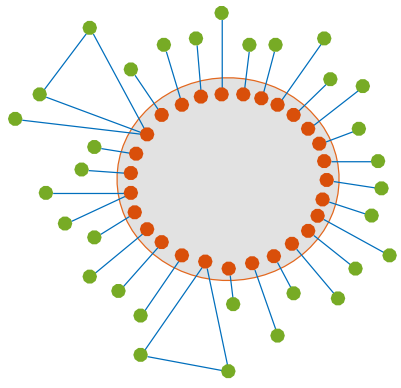

(c) Roman Forum

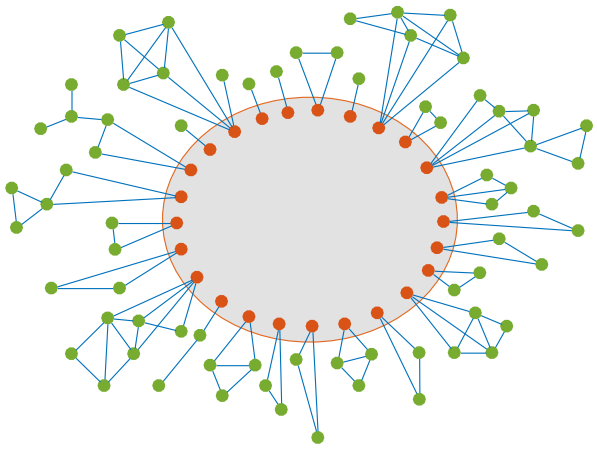

(d) Arts Quad

Figure 12: Graphs corresponding to the Arts Quad dataset [33] and the 1DSfM datasets [45]. The grey circle represents the largest rigid component. Edges not belonging to the largest rigid component are drawn in blue. Nodes which are endpoint of such edges are drawn in red if they belong to the largest rigid component or green if they do not belong to the largest rigid component. All of the remaining nodes/edges are not reported.

Owing to the depth-speed ambiguity, such relative motions are not fully specified: only the direction of relative displacements between pairs of cameras can be measured, for the magnitude is unknown. This means that camera positions are computed from pairwise directions, and this is an instance of bearing-based network localization in 3space, where sensors are the cameras. In this case $\mathcal{G}=(\mathcal{V}, \mathcal{E})$ is known as the epipolar graph [14], [34] or the viewing graph [55], and several approaches can be found in the literature to build it, e.g. [56], [57], [58].

There is a wealth of available approaches in structure from motion literature to recover camera positions, including linear least squares [32], [40], [42], spectral decomposition [30], $\ell_{\infty}$ minimization [34], Riemannian gradient descent [35], the Levenberg-Marquardt algorithm [33], [45], semi-definite programming [19], quadratic programming [36], and the alternating direction method of multipliers [37]. See the survey in [5] for a detailed description of such techniques. However, if bearing-based network localization is ill-posed, then any method will fail to produce a solution. The presence of ill-posed instances in practical scenarios (e.g., the case where the viewing graph is not connected or all the constraints on a node are collinear) is also pointed out in [30] as a "problem pathology". Thus it is important to study the localizability of the problem before solving structure from motion. This is also observed in [19], [36] where the largest rigid component of the epipolar graph is extracted before computing camera positions (using the algorithm described in [21], which is reviewed in Section 2.2.1), which is considered a mandatory part of a global structure-from-motion pipeline.

We report some examples of graphs encountered in real scenarios, which are widely used in structure from motion literature. We consider the Arts Quad dataset [33], which contains 6514 images $^{3}$ of the Arts Quad at Cornell University, and the 1DSfM image collections [45], which contain a number of medium-scale to large-scale Internet datasets ${ }^{4}$. For each dataset, estimates of relative motions between

3. http://vision.soic.indiana.edu/projects/disco/

4. http://www.cs.cornell.edu/projects/1dsfm/ camera pairs are provided, which define an epipolar graph. This graph, however, is not connected in the case of the Arts Quad dataset, thus the largest connected component is considered only, which corresponds to a subset of 5530 nodes and 222044 edges, as detailed in [49]. Note that these edges represent only $2 \%$ of the edges in the complete graph. Table 1 reports this percentage for each dataset, which gives an idea on the sparsity of these graphs.

Table 1: Several statistics are reported for the Arts Quad dataset [33] and the 1DSfM datasets [45]: number of images $n$; percentage of edges; number of articulation points; number of bridges; number of nodes $\bar{n}$ and number of edges $\bar{m}$ that do not belong to the largest rigid component; parallel rigidity index of the largest rigid component $\mathcal{I}_{3}\left(\mathcal{G}^{\prime}\right)$.

\begin{tabular}{lcccccccc}
\hline Dataset & $n$ & $\%$ edges & rigid & articulation & bridges & $\bar{n}$ & $\bar{m}$ & $\mathcal{I}_{3}\left(\mathcal{G}^{\prime}\right)$ \\
\hline Arts Quad & 5530 & 2 & $\boldsymbol{x}$ & 30 & 10 & 70 & 115 & 0 \\
Piccadilly & 2508 & 10 & $\boldsymbol{x}$ & 59 & 62 & 62 & 62 & 0 \\
Roman Forum & 1134 & 11 & $\boldsymbol{x}$ & 28 & 28 & 32 & 34 & 0 \\
Union Square & 930 & 6 & $\boldsymbol{x}$ & 60 & 68 & 77 & 83 & 0 \\
Vienna Cathedral & 918 & 25 & $\boldsymbol{x}$ & 19 & 20 & 20 & 20 & 0 \\
Alamo & 627 & 50 & $\boldsymbol{x}$ & 17 & 19 & 21 & 22 & 0 \\
Notre Dame & 553 & 68 & $\boldsymbol{V}$ & - & - & - & - & 0 \\
Tower of London & 508 & 19 & $\boldsymbol{x}$ & 19 & 19 & 19 & 19 & 0 \\
Montreal N. Dame & 474 & 47 & $\boldsymbol{x}$ & 7 & 7 & 7 & 7 & 0 \\
Yorkminster & 458 & 26 & $\boldsymbol{x}$ & 9 & 10 & 10 & 10 & 0 \\
Madrid Metropolis & 394 & 31 & $\boldsymbol{x}$ & 17 & 15 & 24 & 29 & 0 \\
NYC Library & 376 & 29 & $\boldsymbol{x}$ & 17 & 18 & 18 & 18 & 0 \\
Piazza del Popolo & 354 & 40 & $\boldsymbol{x}$ & 8 & 9 & 9 & 9 & 0 \\
Ellis Island & 247 & 67 & $\boldsymbol{x}$ & 6 & 7 & 7 & 7 & 0 \\
\hline
\end{tabular}

For each dataset, generic parallel rigidity is checked using the randomized test proposed in [19], which computes the rank of the parallel rigidity matrix constructed from a randomly sampled point formation. Note that, as explained in the previous sections, other formulations could be used, such as the equations reported in Section 2.1.1 or the edgebased approach, since they are all equivalent. In the case where a graph is not generically parallel rigid, the largest rigid component $\mathcal{G}^{\prime} \subseteq \mathcal{G}$ is extracted using the algorithm reviewed in Section 2.2.1, as implemented by the authors of [19].

Table 1 reports the outcome of checking parallel rigid- 
ity and, in case of a negative answer, the number of nodes/edges that do not belong to the largest rigid component. The number of articulation points and bridges are also reported, which are found by manual inspection. It is worth noting that only the graph associated with the Notre Dame dataset is generically parallel rigid in 3-space, whereas all of the others are flexible. In particular, the latter exhibit articulation points and bridges, thus they do not satisfy the necessary condition in Proposition 7. Recall that, as a consequence of Corollary 3, the parallel rigidity index of a graph is greater than (or equal to) the number of bridges, thus $\mathcal{I}_{3}(\mathcal{G}) \geqslant 7$ for all the considered datasets except Notre Dame, i.e., the corresponding graphs lie in the bottom (orange) part of Figure 11.

Figure 12 shows a visual representation of the graphs corresponding to four datasets. Due to the inherent difficulty in representing graphs with a high number of nodes, we report a simplified representation where only the edges outside the largest rigid component are drawn. Let us analyse, for instance, the Arts Quad graph reported in Figure 12d: there are 10 bridges and 30 articulation points which make the graph flexible. In particular, 8 bridges correspond to the situation where one camera is linked to just one other camera; 5 articulation points do not belong to the largest rigid component; 25 articulation points belong to the largest rigid component and they link such component to subgraphs of various sizes, including 10 cycles of length 3 .

Table 1 also reports the parallel rigidity index of the largest rigid component of each graph, which is computed using formula (71) with a randomly sampled set of directions. In the case of the Notre Dame dataset, such value coincides with the parallel rigidity index of the initial graph since it is parallel rigid, i.e. $\mathcal{G}^{\prime}=\mathcal{G}$. All of the datasets, restricted to the largest rigid component - which is where the localization problem should be solved - have zero index, thus they provide good graphs for bearing-based localization (in the sense of Section 4.1). In the case of the Notre Dame dataset, this property is satisfied by $\mathcal{G}$ itself, namely the initial graph lies in the upper-right corner of Figure 11.

\section{Conclusion}

In this paper we considered the localizability problem of a sensor network in $d$-space constrained with direction measures, which is studied under the name of parallel rigidity, where global structure from motion is one application in the society of Computer Vision. We provided a unifying view of such a problem: first, we reviewed the node-based formulation of parallel rigidity; then, we described the edgebased formulation, which is equivalent to the node-based one; finally, we suggested how the parallel rigidity index permits to identify which graphs promote error compensation in bearing-based network localization.

As concerns possible future work, several directions could be investigated. First, we aim at establishing if the sufficient conditions in Theorem 10 are also necessary, and, in case of a negative answer, we aim at finding a characterization of localizability in terms of a cycle basis (based on the length of its circuits and how they overlap). Secondly, we will explore under which assumptions (if any) the parallel rigidity index can be rewritten in terms of the generic rank of $C \odot U$. Finally, from the practical perspective, we plan to compare the node-based and edge-based approaches in the presence of noise. In this context, we also aim at studying whether the choice of a particular cycle basis influences the performances of the edge-based localization.

\section{ACKNOWLEDGEMENTS}

The authors would like to thank Beatrice Rossi for her support and valuable discussions on bearing-based localizability that were at the basis of this work.

Federica Arrigoni was partially supported by OP Research, development and education project IMPACT No. CZ.02.1.01/0.0/0.0/15_003/0000468.

\section{REFERENCES}

[1] P. Stoica and K. C. Sharman, "Maximum likelihood methods for direction of arrival estimation," IEEE Transactions on Acoustics, Speech, and Signal Processing, vol. 38, no. 7, pp. 1132 - 1143, 1990.

[2] D. Niculescu and B. Nath, "Ad hoc positioning system (APS) using AOA," in IEEE INFOCOM, 2003, pp. $4104-4113$.

[3] G. Mao, B. Fidan, and B. D. Anderson, "Wireless sensor network localization techniques," Computer Networks, vol. 51, no. 10, pp. $2529-2553,2007$.

[4] R. I. Hartley and A. Zisserman, Multiple View Geometry in Computer Vision, 2nd ed. Cambridge University Press, 2004.

[5] O. Ozyesil, V. Voroninski, R. Basri, and A. Singer, "A survey of structure from motion," Acta Numerica, vol. 26, pp. 305 - 364, 2017.

[6] M. Gavish and A. Weiss, "Performance analysis of bearing-only target location algorithms," IEEE Transactions on Aerospace and Electronic Systems, vol. 28, no. 3, pp. 817 - 828, 1992.

[7] A. N. Bishop, B. D. O. Anderson, B. Fidan, P. N. Pathirana, and G. Mao, "Bearing-only localization using geometrically constrained optimization," IEEE Transactions on Aerospace and Electronics Systems, vol. 45, no. 1, pp. 308 - 320, 2009.

[8] I. Shames, A. N. Bishop, and B. D. O. Anderson, "Analysis of noisy bearing-only network localization," IEEE Transactions on Automatic Control, vol. 58, no. 1, pp. $247-252,2013$.

[9] S. Zhao and D. Zelazo, "Localizability and distributed protocols for bearing-based network localization in arbitrary dimensions," Automatica, vol. 69, pp. $334-341,2016$.

[10] W. Whiteley, "Parallel redrawing of configurations in 3-space," Champlain Regional College, St. Lambert, Quebec, Canada, Tech. Rep., 1986.

[11] _ "Matroids from discrete geometry," in Matroid Theory, ser. AMS Contemporary Mathematics. American Mathematical Society, 1997, pp. 171-313.

[12] R. Connelly, "Generic global rigidity," Discrete and Computational Geometry, vol. 33, no. 4, pp. $549-563,2004$.

[13] B. Servatius and W. Whiteley, "Constraining plane configurations in computer-aided design: combinatorics of directions and lengths," SIAM Journal on Discrete Mathematics, vol. 12, no. 1, pp. $136-153,1999$.

[14] F. Arrigoni, A. Fusiello, and B. Rossi, “On computing the translations norm in the epipolar graph," in Proceedings of the International Conference on 3D Vision (3DV), 2015, pp. 300-308.

[15] T. Eren, W. Whiteley, A. S. Morse, P. N. Belhumeur, and B. D. O. Anderson, "Sensor and network topologies of formations with direction, bearing and angle information between agents," in Proceedings of the IEEE Conference on Decision and Control, 2003, pp. $3064-3069$.

[16] T. Eren, W. Whiteley, and P. N. Belhumeur, "A theoretical analysis of the conditions for unambiguous node localization in sensor networks," Columbia University, Computer Science Department, Tech. Rep. CUCS-O32-M, 2004

[17] —, "Using angle of arrival (bearing) information in network localization," in Proceedings of the IEEE Conference on Decision and Control, 2006, pp. $4676-4681$.

[18] A. Postnikov and A. Spiridonov, "The parallel rigidity index of a graph," 2007, preprint.

[19] O. Ozyesil, A. Singer, and R. Basri, "Stable camera motion estimation using convex programming," SIAM Journal on Imaging Sciences, vol. 8, no. 2, pp. 1220 - 1262, 2015. 
[20] R. Tron, L. Carlone, F. Dellaert, and K. Daniilidis, "Rigid components identification and rigidity enforcement in bearing-only localization using the graph cycle basis," in IEEE American Control Conference, 2015.

[21] R. Kennedy, K. Daniilidis, O. Naroditsky, and C. J. Taylor, "Identifying maximal rigid components in bearing-based localization," in Proceedings of the International Conference on Intelligent Robots and Systems, 2012, pp. $194-201$.

[22] B. Katz, M. Gaertler, and D. Wagner, "Maximum rigid components as means for direction-based localization in sensor networks," in Annual Conference on Current Trends in Theory and Practice of Computer Science, 2007, pp. 330-341.

[23] R. Kennedy and C. J. Taylor, "Network localization from relative bearing measurements," in International Conference on Intelligent Robots and Systems, 2014, pp. $149-156$.

[24] X. Ji and H. Zha, "Sensor positioning in wireless ad-hoc sensor networks using multidimensional scaling," in IEEE INFOCOM, 2004.

[25] P. Biswas, T.-C. Lian, T.-C. Wang, and Y. Ye, "Semidefinite programming based algorithms for sensor network localization," ACM Transactions on Sensor Networks, vol. 2, no. 2, pp. 188-220, 2006.

[26] J. Aspnes, T. Eren, D. Goldenberg, A. Morse, W. Whiteley, Y. Yang, B. Anderson, and P. Belhumeur, "A theory of network localization," IEEE Transactions on Mobile Computing, vol. 5, no. 12, pp. $1663-1678,2006$.

[27] J. M. Hendrickx, B. D. O. Anderson, J.-C. Delvenne, and V. D. Blondel, "Directed graphs for the analysis of rigidity and persistence in autonomous agent systems," International Journal of Robust and Nonlinear Control, vol. 17, no. 10-11, pp. 960-981, 2007.

[28] S. Zhao and D. Zelazo, "Bearing-based formation stabilization with directed interaction topologies," in Proceedings of the IEEE Conference on Decision and Control, 2015.

[29] F. Malapelle, A. Fusiello, B. Rossi, E. Piccinelli, and P. Fragneto, "Uncalibrated dynamic stereo using parallax," in International Symposium on Image and Signal Processing and Analysis (ISPA). IEEE, 2013.

[30] M. Brand, M. Antone, and S. Teller, "Spectral solution of largescale extrinsic camera calibration as a graph embedding problem," in Proceedings of the European Conference on Computer Vision, 2004.

[31] O. Ozyesil, "Camera motion estimation by convex programming," Ph.D. dissertation, Princeton University, 2014.

[32] V. M. Govindu, "Combining two-view constraints for motion estimation," in Proceedings of the IEEE Conference on Computer Vision and Pattern Recognition, 2001.

[33] D. Crandall, A. Owens, N. Snavely, and D. P. Huttenlocher, "Discrete-continuous optimization for large-scale structure from motion," in Proceedings of the IEEE Conference on Computer Vision and Pattern Recognition, 2011, pp. 3001-3008.

[34] P. Moulon, P. Monasse, and R. Marlet, "Global fusion of relative motions for robust, accurate and scalable structure from motion," in Proceedings of the International Conference on Computer Vision, 2013, pp. 3248-3255.

[35] R. Tron and R. Vidal, "Distributed 3-D localization of camera sensor networks from 2-D image measurements," IEEE Transactions on Automatic Control, vol. 59, no. 12, pp. 3325-3340, 2014.

[36] O. Ozyesil and A. Singer, "Robust camera location estimation by convex programming," in Proceedings of the IEEE Conference on Computer Vision and Pattern Recognition, 2015, pp. $2674-2683$.

[37] T. Goldstein, P. Hand, C. Lee, V. Voroninski, and S. Soatto, "ShapeFit and ShapeKick for robust, scalable structure from motion," in Proceedings of the European Conference on Computer Vision, 2016, pp. $289-304$.

[38] D. J. Jacobs and B. Hendrickson, "An algorithm for twodimensional rigidity percolation: the pebble game," Journal of Computational Physics, vol. 137, pp. 346 - 365, 1997.

[39] R. Haas, "Characterizations of arboricity of graphs," Ars Combinatorica, vol. 63, pp. 129 - 137, 2002.

[40] F. Arrigoni, A. Fusiello, and B. Rossi, "Camera motion from group synchronization," in Proceedings of the International Conference on $3 D$ Vision (3DV), 2016, pp. 546-555.

[41] A. Karimian and R. Tron, "Theory and methods for bearing rigidity recovery," in Proceedings of the IEEE Conference on Decision and Control, 2017.

[42] N. Jiang, Z. Cui, and P. Tan, "A global linear method for camera pose registration," in Proceedings of the International Conference on Computer Vision, 2013.
[43] K. Li, Y. Xi, and Y. Zhang, "Some novel characterizations of generic rank of structured matrix," in IEEE American Control Conference, 1998, pp. $2510-2514$.

[44] M. H. Trinh, M.-C. Park, Z. Sun, B. D. O. Anderson, V. H. Pham, and H.-S. Ahn, "Further analysis on graph rigidity," in $c d c, 2016$, pp. $922-927$.

[45] K. Wilson and N. Snavely, "Robust global translations with 1DSfM," in Proceedings of the European Conference on Computer Vision, 2014, pp. 61-75.

[46] V. M. Govindu, "Motion averaging: a framework for efficient and accurate large-scale camera estimation in 3D vision," Tutorial at CVPR, 2017, http://www.ee.iisc.ac.in/labs/cvl/cvpr2017/ tutorial/.

[47] D. Martinec and T. Pajdla, "Robust rotation and translation estimation in multiview reconstruction," in Proceedings of the IEEE Conference on Computer Vision and Pattern Recognition, 2007.

[48] M. Arie-Nachimson, S. Z. Kovalsky, I. Kemelmacher-Shlizerman, A. Singer, and R. Basri, "Global motion estimation from point matches," Proceedings of the Joint 3DIM/3DPVT Conference: $3 D$ Imaging, Modeling, Processing, Visualization and Transmission, 2012.

[49] A. Chatterjee and V. M. Govindu, "Efficient and robust large-scale rotation averaging," in Proceedings of the International Conference on Computer Vision, 2013.

[50] N. Snavely, S. M. Seitz, and R. Szeliski, "Photo tourism: exploring photo collections in 3D," in SIGGRAPH: International Conference on Computer Graphics and Interactive Techniques, 2006, pp. 835-846.

[51] S. Agarwal, N. Snavely, I. Simon, S. M. Seitz, and R. Szeliski, "Building rome in a day," in IEEE International Conference on Computer Vision, 2009.

[52] C. Wu, "Towards linear-time incremental structure from motion," in Proceedings of the International Conference on 3D Vision (3DV). IEEE, 2013.

[53] J. L. Schonberger and J.-M. Frahm, "Structure-from-motion revisited," in Proceedings of the IEEE Conference on Computer Vision and Pattern Recognition, 2016, pp. $4104-4113$.

[54] R. Toldo, R. Gherardi, M. Farenzena, and A. Fusiello, "Hierarchical structure-and-motion recovery from uncalibrated images," Computer Vision and Image Understanding, 2015.

[55] N. Levi and M. Werman, "The viewing graph," in Proceedings of the IEEE Conference on Computer Vision and Pattern Recognition, 2003, pp. $518-522$.

[56] M. Havlena, A. Torii, and T. Pajdla, "Efficient structure from motion by graph optimization," in Proceedings of the European Conference on Computer Vision, 2010, pp. $100-113$.

[57] Y. Lou, N. Snavely, and J. Gehrke, "MatchMiner: Efficient spanning structure mining in large image collections," in Proceedings of the European Conference on Computer Vision, 2012.

[58] T. Shen, S. Zhu, T. Fang, R. Zhang, and L. Quan, "Graph-based consistent matching for structure-from-motion," in Proceedings of the European Conference on Computer Vision, 2016, pp. 139 - 155.

[59] G. Chartrand, Introductory Graph Theory. New York: Dover, 1985.

[60] B. Bollobas, Modern Graph Theory. Springer, 1998.

[61] T. Kavitha, C. Liebchen, K. Mehlhorn, D. Michail, R. Rizzi, T. Ueckerdt, and K. Zweig, "Cycle bases in graphs: Characterization, algorithms, complexity, and applications," Computer Scienze Review, vol. 3, no. 4, pp. 199-243, 2009.

[62] R. E. Tarjan and U. Vishkin, "An efficient parallel biconnectivity algorithm," SIAM Journal on Computing, vol. 14, no. 4, pp. $862-$ 874, 1985.

[63] W. Russel, D. Klein, and J. Hespanha, "Optimal estimation on the graph cycle space," IEEE Transactions on Signal Processing, vol. 59, no. 6, pp. $2834-2846,2011$.

[64] C. Van Loan, "The ubiquitous Kronecker product," J. Comput. Appl. Math, vol. 123, no. 1-2, pp. 85-100, 2000.

[65] C. G. Khatri and C. R. Rao, "Solutions to some functional equations and their applications to characterization of probability distributions," Sankhya: The Indian Journal of Statistics, Series A (19612002), vol. 30, no. 2, pp. pp. 167-180, 1968.

[66] S. Liu and G. Trenkler, "Hadamard, Khatri-Rao, Kronecker and other matrix products," International Journal of Information and Systems Sciences, vol. 4, no. 1, pp. 160 - 177, 2008. 


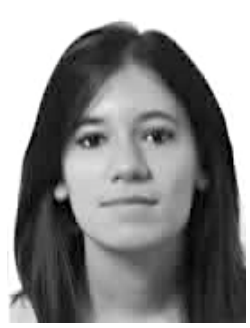

Federica Arrigoni received the M.S. degree in Mathematics from the University of Milan, Italy, in 2013, and the Ph.D. degree in Industrial and Information Engineering from the University of Udine, Italy, in 2018. She is currently a postdoctoral researcher at the Czech Institute of Informatics, Robotics, and Cybernetics of the Czech Technical University in Prague. Her current research focuses on multi-view problems in computer vision, including structure from motion, $3 \mathrm{D}$ registration, and multi-image matching.

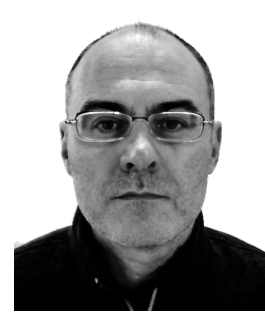

Andrea Fusiello received the Laurea (M.S.) degree in computer science from the University of Udine, Udine, Italy, and the Dottorato di Ricerca (Ph.D.) degree in computer engineering from the University of Trieste, in 1994 and 1999, respectively. He was a Visiting Research Fellow with Heriot-Watt University, Edinburgh, in 1999. From 2001 to 2011, he was with the Department of Computer Science, University of Verona. As an Associate Professor in 2012 he joined the DPIA at the University of Udine, where he is involved in teaching computer vision and computer science basics. His current research interests include computer vision, image analysis, 3-D model acquisition, and image-based rendering. 


\section{APPENDIX A RESULTS FROM GRAPH THEORY}

In this section we review some useful concepts from graph theory. A complete treatment of this subject can be found in [59], [60], [61].

A graph is a pair $\mathcal{G}=(\mathcal{V}, \mathcal{E})$ where $\mathcal{V}$ is a finite set of vertices and $\mathcal{E}$ is a finite set of edges. We use $n$ and $m$ to denote the number of vertices and edges respectively, namely $n=|\mathcal{V}|$ and $m=|\mathcal{E}|$. A weighted graph is a graph together with a weight function $\omega: \mathcal{E} \rightarrow \mathbb{R}^{+}$. If the graph is unweighted, we set $\omega: \mathcal{E} \rightarrow 1$ and call $w$ the uniform weight function. An edge occurring more than once is referred to as a multiple edge, and a graph without multiple edges is called simple. An edge of the form $(v, v)$ is called a loop. In an undirected graph, the degree of a vertex $v$ is the number of times that $v$ occurs as an endpoint of an edge. In a directed graph, the outdegree and indegree of a vertex $v$ are the number of times that $v$ occurs as the tail and head of an edge, respectively.

A subgraph $\mathcal{G}^{\prime}=\left(\mathcal{V}^{\prime}, \mathcal{E}^{\prime}\right)$ of $\mathcal{G}$ is a graph with $\mathcal{V}^{\prime} \subseteq \mathcal{V}$ and $\mathcal{E}^{\prime} \subseteq \mathcal{E}$. If $\mathcal{E}^{\prime}$ is a subset of $\mathcal{E}$, then $\mathcal{G} \backslash \mathcal{E}^{\prime}$ denotes the graph obtained by removing all the edges in $\mathcal{E}^{\prime}$ from $\mathcal{G}$. If $\mathcal{V}^{\prime}$ is a subset of $\mathcal{V}$, then $\mathcal{G} \backslash \mathcal{V}^{\prime}$ denotes the graph obtained by removing all the vertices in $\mathcal{V}^{\prime}$ and their incident edges from $\mathcal{G}$. A path from $v$ to $w$ is a subgraph $\mathcal{G}^{\prime}=\left(\mathcal{V}^{\prime}, \mathcal{E}^{\prime}\right)$ with $\mathcal{V}^{\prime}=\left\{v_{0}=v, v_{1}, \ldots, v_{k}=w\right\}$ and $\mathcal{E}^{\prime}=\left\{\left(v_{0}, v_{1}\right),\left(v_{1}, v_{2}\right), \ldots,\left(v_{k-1}, v_{k}\right)\right\}$. An undirected graph is called connected if there exists a path from each vertex to any other, and a directed graph is called connected if the underlying undirected graph is connected. Any maximal connected subgraph $\mathcal{H}$ is called a connected component. A graph is a tree if it is connected and it has $n-1$ edges. The disjoint union of trees is called a forest. The number of edges in a forest is $n-c c$, where $c c$ denotes the number of connected components in $\mathcal{G}$. A subgraph $\mathcal{G}^{\prime}$ of a connected graph $\mathcal{G}$ is called a spanning tree if it has the same vertices of $\mathcal{G}$ and it is a tree. If $\mathcal{G}$ is not connected, any union of spanning trees for each connected component is called a spanning forest.

A connected graph $\mathcal{G}$ is called biconnected if it has no articulation points, where a vertex $v \in \mathcal{V}$ is an articulation point (or cut vertex) if $\mathcal{G} \backslash\{v\}$ is not connected. Any maximal biconnected subgraph is called a biconnected component. Equivalently, a biconnected component is a maximal set of edges such that any two edges in the set lie on a common circuit. It can be shown that biconnected components partition the edges of the graph [62], where a single edge is considered biconnected by definition. However, they may share vertices with each other.

A connected graph $\mathcal{G}$ is called bridgeless if it has no bridges, where an edge $e \in \mathcal{E}$ is a bridge (or cut edge) if $\mathcal{G} \backslash\{e\}$ is not connected. It can be shown (e.g. [59]) that if $\mathcal{G}$ is a connected graph on at least 3 vertices and $e$ is a bridge, then $e$ is incident to (at least) one articulation point. In other words, if $\mathcal{G}$ is biconnected and contains at least 3 vertices, then it is bridgeless.

\section{A.1 Cycle Bases}

Let $\mathbb{K}$ be a field. A cycle in a graph $\mathcal{G}$ is a vector $\mathbf{c} \in \mathbb{K}^{m}$ such that for any vertex $v \in \mathcal{V}$ it holds

$$
\sum_{e \in \delta_{+}(v)}[\mathbf{c}]_{e}=\sum_{e \in \delta_{-}(v)}[\mathbf{c}]_{e}
$$

where $\delta_{+}(v)$ and $\delta_{+}(v)$ denote the edges leaving and entering $v$, respectively, and $[\mathbf{c}]_{e}$ denotes the component of $\mathbf{c}$ indexed by edge $e$. It is shown in [59] that an edge of a connected graph is a bridge if and only if it does not belong to any cycle.

A cycle is simple if $[\mathbf{c}]_{e} \in\{-1,0,1\}$ for all $e \in \mathcal{E}$, and a simple cycle is a circuit if its support (i.e. the set of edges with $[\mathbf{c}]_{e} \neq 0$ ) is connected and for any vertex $v \in \mathcal{V}$ there are at most two edges in the support incident to $v$. The set of cycles forms a vector space over $\mathbb{K}$, which is called the cycle space of $\mathcal{G}$, and a cycle basis is a set of circuits forming a basis of such a space. It can be shown [60], [61] that if $\mathcal{G}$ is connected the dimension of the cycle space is given by the cyclomatic number

$$
\nu=m-n+1 \text {. }
$$

If $\mathcal{T}$ is a spanning tree of $\mathcal{G}$, then adding any edge from $\mathcal{E} \backslash \mathcal{T}$ to $\mathcal{T}$ generates a circuit [61]. The set of such circuits forms a cycle basis, which is referred to as the fundamental cycle basis. Figure 13 reports one example.

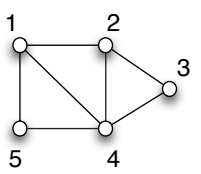

(a)

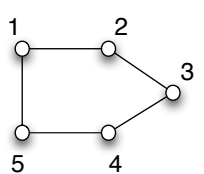

5

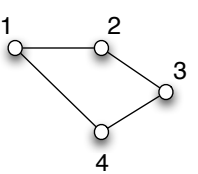

(b)
Figure 13: Left: graph with $n=5$ nodes and $m=7$ edges. Right: fundamental cycle basis associated with the spanning tree $\mathcal{T}=\{(1,2),(2,3),(3,4),(4,5)\}$.

Particularly interesting are the cases $\mathbb{K}=\mathbb{Q}$ and $\mathbb{K}=$ $\mathbb{Z}_{2}$, which correspond to a directed and undirected graph, respectively. If $\mathbb{K}=\mathbb{Q}$, the field of rationals, then the cycle basis is referred to as the directed cycle basis. Directed cycles may use arcs in forward $\left([\mathbf{c}]_{e}>0\right)$ or backward $\left([\mathbf{c}]_{e}<\right.$ 0 ) direction. A directed cycle basis $\left\{\mathbf{c}_{1}, \mathbf{c}_{2}, \ldots, \mathbf{c}_{\nu}\right\}$ is called a/an

- integral cycle basis if each cycle $\mathbf{c}$ of $\mathcal{G}$ can be written as an integer linear combination of the circuits in the basis, namely

$$
\exists \lambda_{i} \in \mathbb{Z}: \mathbf{c}=\lambda_{1} \mathbf{c}_{1}+\lambda_{2} \mathbf{c}_{2}+\ldots \lambda_{\nu} \mathbf{c}_{\nu}
$$

- zero-one cycle basis (or totally unimodular cycle basis) if each cycle $\mathbf{c}$ of $\mathcal{G}$ can be written as a linear combination with coefficients in $\{-1,0,+1\}$ of the circuits in the basis, namely

$$
\exists \lambda_{i} \in\{-1,0,+1\}: \mathbf{c}=\lambda_{1} \mathbf{c}_{1}+\lambda_{2} \mathbf{c}_{2}+\ldots \lambda_{\nu} \mathbf{c}_{\nu} .
$$

If $\mathbb{K}=\mathbb{Z}_{2}=G F(2)$, the field of two elements, then the cycle basis is referred to as the undirected cycle basis. In $\mathbb{Z}_{2}$ the only non-zero element in the field is $-1=+1$, thus a 


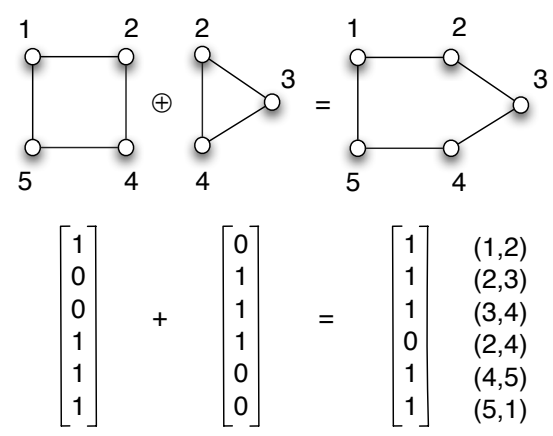

Figure 14: Sum of two cycles.

cycle is a vector $\mathbf{c} \in \mathbb{Z}_{2}^{m}$ such that for any vertex $v \in \mathcal{V}$ it holds

$$
\sum_{e \in \delta(v)}[\mathbf{c}]_{e}=0
$$

where $\delta(v)$ denotes the set of edges incident to $v$. Alternatively, an undirected cycle can be viewed as a set of edges, namely it is a subgraph in which every vertex has even degree. The sum of two cycles, denoted by $\oplus$, is a cycle where the common edges vanish, and, more generally, the sum of cycles is the cycle consisting of all the edges that are contained in an odd number in the addends. This concept is illustrated in Figure 14. It can be shown that an undirected cycle basis can be turned into a directed cycle basis, but the converse is not true [61].

The relationships between the aforementioned classes of cycle bases are illustrated in Figure 15. The proofs of such inclusions are provided in [61], which also reports counterexamples showing that the inclusions are strict. Note that any directed graph has a basis of each type, since any directed graph has a fundamental cycle basis, and all the other classes generalize fundamental cycle bases.

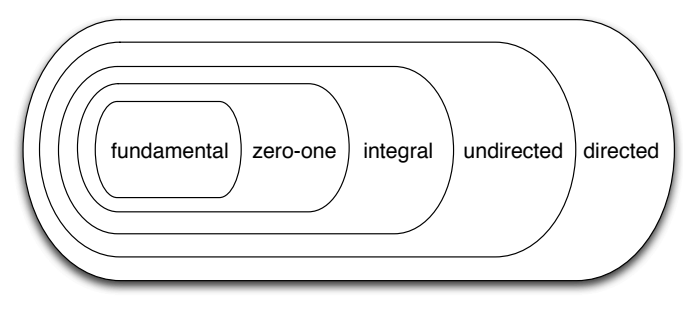

Figure 15: Classification of cycle bases.

\section{A.2 Matrices associated with graphs}

The adjacency matrix $A$ of a graph $\mathcal{G}$ is the $n \times n$ matrix whose elements indicate whether pairs of vertices are adjacent or not, namely

$$
[A]_{i, j}= \begin{cases}1 & \text { if }(i, j) \in \mathcal{E} \\ 0 & \text { otherwise }\end{cases}
$$

If $\mathcal{G}$ does not contain loops, then $A$ has zero diagonal. Note that the adjacency matrix is symmetric if the graph is undirected.
The incidence matrix $B$ of a directed graph $\mathcal{G}$ is the $n \times m$ matrix defined by

$$
[B]_{k, e}= \begin{cases}1 & \text { if } k \text { is the head of edge } e \\ -1 & \text { if } k \text { is the tail of edge } e \\ 0 & \text { otherwise }\end{cases}
$$

The rows of $B$ correspond to vertices and the columns correspond to edges. Note that each column has exactly two non zero entries, which correspond to the endpoints of the edge associated with that column. The incidence matrix $B$ of an undirected graph $\mathcal{G}$ is defined considering a particular orientation of the edges. It is shown in [60] that, if $\mathcal{G}$ is connected, then

$$
\operatorname{rank}(B)=n-1 \text {. }
$$

The degree matrix $D$ of an undirected graph $\mathcal{G}$ is the $n \times n$ diagonal matrix such that $[D]_{i, i}$ contains the degree of node $i$. Equivalently, it can be defined as

$$
D=\operatorname{diag}\left(A \mathbf{1}_{n \times 1}\right)
$$

where $\mathbf{1}_{n \times 1}$ denotes a $n \times 1$ matrix filled by ones, thus $A \mathbf{1}_{n \times 1}$ is the sum of the rows of $A$. In the case of a directed graph, either the indegree or the outdegree can be used. The Laplacian matrix $L$ is defined as

$$
L=D-A \text {. }
$$

It can be checked that, independently of the orientation of the edges, the following equation holds for an undirected graph

$$
L=B B^{\top}
$$

which implies that $L$ is symmetric and positive semidefinite, and, if the graph is connected, $\operatorname{rank}(L)=\operatorname{rank}(B)=n-1$.

The notion of adjacency matrix can be extended to the case of a weighted graph, which translates in letting the entries of $A$ to assume non-negative values. Specifically, $[A]_{i, j}$ contains the weight of edge $(i, j)$, and $[A]_{i, j}=0$ still indicates that $(i, j) \notin \mathcal{E}$. In this case Equations (82) and (83) still make sense, which define the degree matrix and Laplacian matrix of a weighted graph, respectively.

The cycle matrix $C$ corresponding to a cycle basis of a connected graph $\mathcal{G}$ is the $(m-n+1) \times m$ matrix having the incidence vectors of the circuits in the basis in its rows. Note that the cycle matrix has columns of zeros in correspondence of bridges (if they exist). The following equation [60], [63] expresses the relation between the cycle matrix and the incidence matrix

$$
C B^{\top}=0
$$

\section{APPENDIX B KRONECKER AND KHATRI-RAO PRODUCTS}

This appendix is devoted to the Kronecker and Khatri-Rao products [64], [65], [66], which are widely used in this paper.

Let $A$ and $B$ be two real matrices of dimension $m \times r$ and $n \times s$ respectively. The Kronecker product of $A$ and $B$ [64], denoted by $A \otimes B$, is defined as

$$
A \otimes B=\left[\begin{array}{cccc}
{[A]_{1,1} B} & {[A]_{1,2} B} & \ldots & {[A]_{1, r} B} \\
{[A]_{2,1} B} & {[A]_{2,2} B} & \ldots & {[A]_{2, r} B} \\
\ldots & & & \ldots \\
{[A]_{m, 1} B} & {[A]_{m, 2} B} & \ldots & {[A]_{m, r} B}
\end{array}\right]
$$


where each $[A]_{i, j} B$ is a block of dimension $n \times s$, thus $A \otimes B$ has dimension $m n \times r s$. The Kronecker product is associative, distributive (with respect to the sum of matrices), but not commutative, and it satisfies the following properties

$$
\begin{gathered}
(A \otimes B)^{\top}=A^{\top} \otimes B^{\top} \\
(A \otimes B)^{-1}=A^{-1} \otimes B^{-1} \\
(A \otimes B)(C \otimes D)=(A C) \otimes(B D) \\
\operatorname{vec}(A X B)=\left(B^{\top} \otimes A\right) \operatorname{vec}(X)
\end{gathered}
$$

where $\operatorname{vec}(\cdot)$ denotes the vectorization operator which transforms a matrix into a vector by stacking the columns of the matrix one underneath the other.

Let $A=U_{A} \Sigma_{A} V_{A}^{\top}$ and $B=U_{B} \Sigma_{B} V_{B}^{\top}$ be the singular value decompositions of $A$ and $B$, respectively, then

$$
A \otimes B=\left(U_{A} \otimes U_{B}\right)\left(\Sigma_{A} \otimes \Sigma_{B}\right)\left(V_{A} \otimes V_{B}\right)^{\top}
$$

which implies

$$
\operatorname{rank}(A \otimes B)=\operatorname{rank}(A) \operatorname{rank}(B) .
$$

Thus the Kronecker product of two matrices is invertible if and only if both the factors are invertible.

Consider now two real matrices $A$ and $B$ of dimension $m \times r$ and $n \times r$ respectively, and denote the columns of $A$ by $\mathbf{a}_{1}, \ldots, \mathbf{a}_{r}$ and those of $B$ by $\mathbf{b}_{1}, \ldots, \mathbf{b}_{r}$. The Khatri-Rao product of $A$ and $B$ [65], [66], denoted by $A \odot B$, is defined as

$$
A \odot B=\left[\begin{array}{llll}
\mathbf{a}_{1} \otimes \mathbf{b}_{1} & \mathbf{a}_{2} \otimes \mathbf{b}_{2} & \cdots & \mathbf{a}_{r} \otimes \mathbf{b}_{r}
\end{array}\right]
$$

where each $\mathbf{a}_{i} \otimes \mathbf{b}_{i}$ is a vector of dimension $m n$, thus $A \odot B$ has dimension $m n \times r$. The Khatri-Rao product is associative, distributive, but not commutative, and it satisfies the following equalities

$$
\begin{gathered}
(A \otimes B)(C \odot D)=(A C) \odot(B D) \\
\operatorname{vec}(A \operatorname{diag}(\mathbf{x}) B)=\left(B^{\top} \odot A\right) \mathbf{x}
\end{gathered}
$$

where $\operatorname{diag}(\mathbf{x})$ transforms the vector $\mathbf{x}=\left[\begin{array}{lll}x_{1} & \ldots & x_{r}\end{array}\right]^{\top}$ into a diagonal matrix with elements $x_{1}, \ldots, x_{r}$ along the diagonal. 